\title{
Does blockchain patent-development influence Bitcoin risk?
}

\author{
Yang $\mathrm{Hu}^{a}$, Yang (Greg) $\mathrm{Hou}^{a}$, Les Oxley ${ }^{a}$, Shaen Corbet ${ }^{a, b *}$ \\ ${ }^{a}$ School of Accounting, Finance and Economics, University of Waikato, New Zealand \\ ${ }^{b}$ DCU Business School, Dublin City University, Dublin 9, Ireland \\ * Corresponding Author: shaen.corbet@dcu.ie
}

\begin{abstract}
The paper presents a novel analysis specifically investigating as to whether stocks associated with leading blockchain patent-developments influence the price volatility of Bitcoin across multiple time frequencies. It is important to further develop our understanding of the inter-dynamics between this relatively youthful financial product and pricing sensitivities associated with corporate technological advancement. Several interesting results are presented. First, Bitcoin is identified as a volatility receiver instead of a transmitter across all of the time frequencies considered during periods of patent development. Secondly, Microsoft, Mastercard, Intel and Visa contribute the largest volatility spillovers to the Bitcoin market due to patent development. Finally, for most of the companies considered, the calculated spillover effects towards Bitcoin markets are found to increase from the short-term to the long-term. These results suggest the existence of an avenue through which large corporations can influence cryptocurrency prices through their announcements of future technological intentions. The inherent risks incorporated with blockchain and cryptocurrency patent-development should be studied in detail, with particular warnings presented to those companies with no evidence of prior exposure and market knowledge.
\end{abstract}

Keywords: Bitcoin; Cryptocurrency; Blockchain; Volatility; Spillovers; Contagion; Patents; Intellectual Property.

\section{Introduction}

One of the most well-known and established features of Bitcoin price-dynamics surrounds the exceptional levels of price volatility inherent across all exchanges on which the new digital currency trades. Several studies have recently attempted to examine the return and volatility transmissions between Bitcoin or other cryptocurrency and a variety of other financial assets (Symitsi and Chalvatzis [2018], Yi et al. [2018], Corbet et al. [2018a] and Koutmos [2018]). However, none of this research to date has considered the associated volatility transmissions between the stock prices of multinational companies who have submitted patents, and the subsequent interactions between 
such decision-making and the prices of cryptocurrencies. It is entirely possible that such intention to further this growing technological market could be observed by the wider market to be a reaffirming signal of future stability. However, companies that have announced their intentions to enter blockchain and cryptocurrency markets, has also raised some elements of doubt as to the true underlying intentions to further technological progress, which has in some cases been identified as a somewhat cynical, and most likely to be taking advantage of recent cryptocurrency price appreciation rather than further advancement of the developing sector (Corbet et al. [2019, 2020]). The analysis of patent development within the blockchain and cryptocurrency sector provides a novel mechanism through which we can identify new sectoral entrants. It is also of concern that the incorporation of such new technological products can also manifest in substantially elevated corporate risks, particularly through the incorporation of digital currency risk to which such companies could have little or no experience in hedging (Akhtaruzzaman et al. [2019], Corbet et al. [2019]). Such intentions would be widely observed as a significant threat upon cryptocurrency markets, in particular the largest cryptocurrency among them, namely Bitcoin.

The very use of patents to inherently protect the value of innovation is of the utmost importance, providing protection of the corporate entity's intellectual property, while providing the legal right to exclude others from making, using, selling and importing an invention for a limited period of time. While the decision of large multinational corporations to enter into development processes within cryptocurrency markets is a strong signal of the continued development of the product, it is important that they do so conditionally, and respectively, of the culminating risks that are inherent in these products (Corbet et al. [2019]). The transfer of risk is one of the central issues that has eroded trust in the development of cryptocurrencies to date, with illicit and questionable ethical behaviour accounting for a number of broad concerns within these rapidly growing exchanges. The inherent development of cybercriminality within cryptocurrency markets has generated substantial concern. Gandal et al. [2018] identified the impact of suspicious trading activity on the Mt. Gox Bitcoin currency exchange, in which approximately 600,000 Bitcoin were fraudulently acquired. The research concludes through the condition that suspicious trading activity likely caused the unprecedented spike in the USD-BTC exchange rate in late 2013, when the rate jumped from around $\$ 150$ to more than $\$ 1,000$. Further, Foley et al. [2019] estimated that around $46 \%$ of Bitcoin transaction were associated with illicit activity each year, suggesting that cryptocurrencies are directly responsible for a rapid transformation of the black markets by enabling black e-commerce, while Corbet et al. [2019] found that cryptocurrency cybercriminality events increase both the price volatility of the targeted cryptocurrency and broad cross-cryptocurrency correlations. Wei [2018a] identified that an increase in Tether trading following a subsequent decrease in Bitcoin returns, while further identifying the generation of abnormal corporate returns. This sentiment is echoed by Cahill et al. [2020] investigated the price reaction of 713 listed companies in response to blockchain-related announcements, finding evidence of abnormal returns being directly linked to 
the performance of Bitcoin. Speculative announcements exhibit higher returns than non-speculative announcements, while the authors hypothesise that investors have confused Bitcoin and blockchain, and used the performance of bitcoin as an indicator of the expected success of the newly developed blockchain technology. Further fears have surrounded the continued escalation of energy usage in the mining processes of cryptocurrencies. Further, Corbet et al. [2019] identified a sharp and sustained increase in both the share price and price volatility of Kodak after the KODAKCoin announcement, which was the decision for Kodak to incorporate blockchain technology, leading to an increased correlation between the price of Kodak shares and Bitcoin. Das and Dutta [2020] reported a negative relationship between Bitcoin's energy consumption and miner's revenue using a quantile and Markov regime switching regression, identifying that it would not be viable to sustain the business unless cheap energy sources and efficient mining hardware are relied upon.

While considering the above effects that the erosion of trust has continued to play within cryptocurrency markets, this research focuses on the provision of support through the growth in patents and research by major corporations within the growing digital currency sector. The continued provision of support by major corporations will further entice others to develop research through direct reputational benefits and enhancement, but also the elevated threat of potential corporate technological advancement, which, if competitors do not manage could lead to significant corporate advancement. Specifically, this study explores volatility spillovers between Bitcoin prices and these identified blockchain patent-related stocks using the time and frequency domains approaches of Diebold and Yilmaz [2012] and Baruník and Křehlík [2018]. The paper attempts to answer the following three questions: 1) is Bitcoin a volatility transmitter or receiver of such patent-related volatility? 2) if Bitcoin is a volatility receiver, which stocks have been found to contribute the most to the volatility of Bitcoin? and finally 3 ) do the volatility spillover effects vary from short to long terms? Such an analysis bears substantial benefit for policy-makers and regulators. It is very much of interest to develop our understanding of the mechanisms through which cryptocurrency volatility is sourced. Further, it is important to understand as to whether a market of such rapidly development is both aware and exposed to the threat of new market entrants. This paper explicitly contributes to the literature in three ways. First, we study, for the first time, the static and dynamic volatility spillovers between top ranked blockchain patent-related stocks and Bitcoin prices. Second, we investigate the role of volatility transmission among blockchain patent holders for Bitcoin market at different time frequencies. Third, we explore as to which company plays the most important role in driving the volatility of Bitcoin to its all-time high in December 2017.

The remainder of this paper is organised as follows. Section 2 outlines the key previous literature associated the understanding and development of cryptocurrency risk, with emphasis on Bitcoin in isolation. Section 3 describes the data and econometric methods. Section 4 presents the empirical results and Section 5 concludes and discusses the implications. 


\section{Previous Literature}

While Corbet et al. [2019] provide a concise systematic analysis of the available literature on cryptocurrency research to date, they note the distinct breadth of research on market volatility and liquidity in comparison to the relatively coverage of sparse of associated regulatory and legislative issues surrounding the rapid growth of these new financial products. Dwyer [2015] explained how the use of these technologies and limitation of the quantity produced can create an equilibrium in which a digital currency has a positive value. A substantial debate continues to surround as to what exactly one Bitcoin represents when considering traditional financial assets. Baur et al. [2018] found that the asset is mainly used as a speculative investment and not as an alternative currency and medium of exchange. Further, Corbet et al. [2019] concluded that Bitcoin is a speculative asset rather than a currency and is not altered by the introduction of futures trading, while Ammous [2018] identified it's sole potential to serve as a store of value, due to its strict commitment to low supply growth, credibly backed by the network's distributed protocol. Bohme et al. [2015] considered some of the regulatory issues facing broad market participants, while Ahluwalia et al. [2020] focused on sectoral start-up financing.

While many corporations with no previous history or experience with digital currency continue to search for avenues of development in this new expanding technological sector, it is very much of concern that such expansion could enable a pathway for risk and contagion effects to enter, to which the company and its investors could be ill-equipped to manage. To this effect, is important to consider research surrounding the stylised facts associated with cryptocurrency market volatility and their broad relationship with other financial markets. Alexander and Dakos [2020], Celeste et al. [2019] and Zhang et al. [2019] focused on the stylised facts and differentials based on high-frequency cryptocurrency data while Chu et al. [2020] identified a momentum strategy to be used successfully for cryptocurrency trading in a high frequency setting. Aharon and Qadan [2019] and Eross et al. [2019] analysed intra-day and day-of-the-week pricing dynamics, while Alexander and Dakos [2020], Ibikunle et al. [2020], Brandvold et al. [2015] and Zeng et al. [2019] focused on the price discovery and information flow processes within cryptocurrency markets. Hu et al. [2019] investigated the interaction between these markets and the efficient market hypothesis, identifying no empirical support and concluding that there exists substantial market inefficiency in cryptocurrencies. Wei [2018b] identified a strong relationship between the Hurst exponent and liquidity on a cross-sectional basis, finding that liquidity plays a significant role in market efficiency and return predictability of new cryptocurrencies, while Matkovskyy [2019] found that trading increases as prices decrease, demonstrating participants' lack of confidence and consensus in a price-jump period..

When focusing on cryptocurrency volatility and contagion dynamics, Katsiampa et al. [2019] identified that when analysing cryptocurrency returns, that all conditional variances are significantly affected by both previous squared errors and past conditional volatility, while Antonakakis et al. [2019] find evidence that high cryptocurrency market uncertainty correspond to strong connected- 
ness and vice versa and Ciaian et al. [2018] identified that the Bitcoin-altcoin price relationship is significantly stronger in the short-run than in the long-run. Alvarez-Ramirez et al. [2018] found that Bitcoin-altcoin price relationship is significantly stronger in the short-run than in the long-run. Ji et al. [2019] found that Litecoin and Bitcoin are at the centre of the connected network of returns when analysing the dynamic connectedness of cryptocurrency markets. Further relationships have been identified between cryptocurrencies and implied volatility (Akyildirim et al. [2019]; Corbet et al. [2018b]), monetary policy decision-making (Meegan et al. [2018]; Corbet et al. [2020]), fundamental laws (Kristoufek [2019]), and futures and derivatives markets (Corbet et al. [2018]). With regards to portfolio design, both Briere et al. [2015] and Akhtaruzzaman et al. [2019] identify lower dynamic conditional correlations between Bitcoin and industry portfolios and bond index, allowing an investment in Bitcoin to hedge the risk against industry portfolios and bonds.

Further evidence of the risks surrounding the decision to incorporate cryptocurrency and blockchain into the corporate process surrounds that research that has focused on the bubble-dynamics in cryptocurrency markets. Cheung et al. [2015] identified three large bubbles in the latter part of the period 2011-2013 and during the demise of the Mt Gox exchange. This result was echoed by Chaim and Laurini [2019], Geuder et al. [2019] and Cretarola and Figà-Talamanca [2019]. Further, Corbet et al. [2018] similarly identified the presence of bubbles in Bitcoin markets again in the period after that considered by Cheung et al. [2015]. Cagli [2019] investigated the explosive behaviour found within cryptocurrency markets to find that other than NEM, all tested assets experienced explosive behaviour and significant pairwise co-movement relationships. Xiong et al. [2019] attempted to include the cost of production of cryptocurrency into such analysis of potential bubbles in Bitcoin pricing, identifying a high-risk period in late-2020 as output declines. Cheng et al. [2019] identified a sharp increase in the number of initial 8-K disclosures of Blockchain by firms as the price of the assets began to appreciate substantially, which is found to be directly related to a positive investor reaction within the following seven days, which subsequently reverses in the month thereafter. As research continues to develop, it is still important to consider the early work of Cheah and Fry [2015] who were amongst the first to identify a bubble in Bitcoin, but more alarmingly stated that evidence at the time suggested that the fundamental price of Bitcoin was zero.

When analysing Bitcoin, Katsiampa [2017] found that the AR-CGARCH model was the optimal conditional heteroskedasticity methodology when considering goodness-of-fit. Balcilar et al. [2017] found using causality-in-quantiles testing that volume can predict returns with the exception of Bitcoin during bear and bull market regimes. Yi et al. [2018] analysed fifty-two separate cryptocurrencies to find that the largest cryptocurrencies are more likely to propagate volatility shocks to others. Further, Jin et al. [2019] use a multifractal detrended cross-correlation analysis (MF-DCCA) to find that multifractality exists in the cross-correlations among the three hedging assets, namely Bitcoin, oil and gold, where Bitcoin is found to be more susceptible to price fluctuations from gold and crude oil markets. The DCC-MVGARCH methodology is found to indicate that the spillover 
effects from gold and crude oil markets to Bitcoin market are much stronger than other spillovers.

As observed, much research focused on the influence of cryptocurrency pricing on that of the corporate entity. However, to date, no research has focused on the fundamental response of cryptocurrency markets to the most-likely positive news that a reputable, large market-capitalisation company has taken the decision to enter and attempt to further advance blockchain and cryptocurrencydevelopment. Such a development would most likely be observed as both reassuring and reaffirming of the continued presence of blockchain and cryptocurrencies. The value of such development has been central to the work of Chen et al. [2019], who used data on patent filings from 2003 to 2017, and applied machine learning to identify to classify innovations by their underlying technologies, indicating that most FinTech innovations yield substantial returns with blockchain being the most rewarding. To raise capital for such ventures, Fisch [2019] found that technical white papers and high-quality source codes increase the amount raised, while patents are not associated with increased amounts of funding. Pan et al. [2018] examined how exploitative technological diversification (ETD) affects firm performance and what factors may moderate this relationship using patent data for 1,569 Chinese listed firms with 7,555 observations from 2003 to 2014 to find that the relationship between ETD and firm performance is inversely U-shaped. Lin [2015] found that firms headquartered near a knowledge centre experience higher market-to-book ratios and higher stock volatility. Vitt and Xiong [2016] analysed as to whether the relationship between the patent activities of high-tech companies and the dynamics of their stock price movement. The results indicate the presence of a significant impact of patent activity on stock movement and on its essential statistical characteristics of drift and volatility. Bartram et al. [2012] found that stock volatility in the United States is significantly higher as it increases with investor protection, stock market development, new patents, and firm-level investment in R\&D. Chow and Fung [2008] proposed a model identifying stock price volatility and variations in innovation effort using a Multivariate GARCH structure designed to extract information for risk prediction using patent data for US computer firms.

\section{Data and Methodology}

\subsection{Data}

Primarily, we decided as to which blockchain patent-related share prices should be included in our analysis. Based on the number of blockchain patents from three ranking lists, we select fourteen publicly-listed firms including IBM, Intel, Bank of America (BofA), Mastercard, Visa, Microsoft, Walmart, British Telecom (BT), Accenture, Ping An Insurance (Ping An), Baidu, Tencent, JD.com (JD) and Alibaba. ${ }^{1}$. Both the US and China are world leaders in the development of blockchain

\footnotetext{
${ }^{1}$ We select the blockchain patent-related companies from three rankings: NASDAQ; Statista; and IPlytics
} 
technologies and the above selected companies can be divided into two main groups: US firms (IBM, Intel, BOfA, Mastercard, Visa, Microsoft and Walmart) and Chinese firms (Ping An, Baidu, Tencent, JD, and Alibaba). In addition, Accenture is an Irish-domiciled multinational professional services company that provides services in strategy, consulting, digital, technology and operations.

We next consider the starting date for these blockchain patent holding companies for our empirical analysis. One selection mechanism surrounds the identification of the number of blockchain patent applications, which is presented as Figure 1 for the period between 2008 and 2019. From this figure, there is evidence of a sharp increase in the submitted applications in 2014. As the application process takes some time, it is reasonable to believe that the effects on the performance of the corresponding stocks are likely long-term. Hence, we decide to consider the performance of blockchain patent-related stock prices over the last five years, selecting 2014 as the most effective starting point to adequately reflect the major development in blockchain technologies. We then proceed to obtain the daily high and low prices for each company from Thomson Reuters Datastream between 10 November 2014 and 07 November 2019, presenting a robust dataset consisting of 1,142 observations. In addition, we also use the daily high and low prices of Bitstamp's Bitcoin prices from the same source.

\section{Insert Figure 1 about here}

We then follow Diebold and Yilmaz [2012] to calculate volatility as Equation 2:

$$
\begin{aligned}
\tilde{\sigma}_{i t}^{2} & =0.361\left[\ln \left(P_{\mathrm{it}}^{\mathrm{max}}\right)-\ln \left(P_{\mathrm{it}}^{\mathrm{low}}\right)\right]^{2}, \\
\hat{\sigma}_{i t} & =100 \sqrt{365 \tilde{\sigma}_{i t}^{2}},
\end{aligned}
$$

where $\tilde{\sigma}_{i t}^{2}$ is daily variance using daily high and low prices and $\hat{\sigma}_{i t}$ represents the corresponding estimate of volatility of the annualised daily percent standard deviation (volatility). ${ }^{2}$

\section{Insert Figure 2 about here}

The volatilities calculated based on Equation 2 for Bitcoin and each company are presented as Figure 2. First, as can be seen from this figure, Bitcoin had the largest volatility at the end of March, 2016. Second, most companies experienced a large spike in volatility during the third quarter of 2015 (e.g., Intel, BofA, Mastercard, Visa, Microsoft, Walmart, Accenture, Ping An, Baidu, Tencent, JD and Alibaba). We also present descriptive statistics for the volatility data in

\footnotetext{
${ }^{2}$ As Bitcoin market is open $24 / 7$, it is not suitable to estimate volatility using the open and close prices. Therefore, we use the high and low prices to estimate volatility.
} 
Table 1. Among all volatility series, Bitcoin has the highest mean and maximum. Three Chinese firms (e.g., Ping An, Baidu and Alibaba) have relatively high means compared with those of other companies except Bitcoin.

\section{Insert Table 1 about here}

\subsection{Methodology}

We apply a spillover measure based on forecast error variance decompositions from a generalized VAR framework of Diebold and Yilmaz [2012] and a frequency-dependent measure based on a spectral representation of variance decomposition approach of Baruník and Křehlík [2018] to study the strength of spillovers for the variables of interest. In this paper, we refer to the Diebold and Yilmaz [2012] and Baruník and Křehlík [2018] approaches as DY12 and BK18. The DY12 approach measures total, directional, net and pairwise spillover indexes while the BK18 procedure further provides a measure of these spillover indexes in short-, medium-, and long-term frequency by taking into account the different strengths of the shocks to economic variables at different frequencies. The following section aligns with Baruník and Křehlík [2018]. Let us consider a covariance stationary $N$-variable $\operatorname{VAR}(p), \mathbf{x}_{t}=\left(x_{t, 1}, \ldots, x_{t, N}\right)$, by a VAR model of order $p$ as:

$$
\mathbf{x}_{t}=\Phi_{1} \mathbf{x}_{t-1}+\Phi_{2} \mathbf{x}_{t-2}+\cdots+\Phi_{p} \mathbf{x}_{t-p}+\boldsymbol{\epsilon}_{t}
$$

where $\Phi_{1}, \ldots, \Phi_{p}$ are coefficient matrices and $\boldsymbol{\epsilon}_{t}$ is a white noise covariance matrix $\boldsymbol{\Sigma}$. Or we can re-write Equation 3 as:

$$
\mathbf{x}_{t}=\mathbf{\Psi}(L) \boldsymbol{\epsilon}_{t}
$$

where $\boldsymbol{\Psi}(L)=$ is an infinite $N \times N$ matrix lag-polynomial.

Variance decomposition provide useful information about how much of the future uncertainty of variable $j$ is due to shocks in variable $k$. The identification approach of Cholesky factorization depends on the order of the variables. The generalized VAR identification scheme of Pesaran and Shin [1998] produces variance decompositions invariant to the orders of the variables.

The generalized variance decompositions can be written as follows:

$$
\left(\boldsymbol{\theta}_{H}\right)_{j, k}=\frac{\left.\sigma_{k k}^{-1} \sum_{h=0}^{H}\left(\boldsymbol{\Psi}_{h} \boldsymbol{\Sigma}\right)_{j, k}\right)^{2}}{\sum_{h=0}^{H}\left(\boldsymbol{\Psi}_{h} \boldsymbol{\Sigma} \boldsymbol{\Psi}_{h}^{\prime}\right)_{j, j}}
$$

where $\boldsymbol{\Psi}_{h}$ is a $(\mathrm{N} \times \mathrm{N})$ matrix of coefficients at lag $h$ and $\sigma_{k k}=(\boldsymbol{\Sigma})_{k, k}$. The generalized variance decomposition $\left(\boldsymbol{\theta}_{H}\right)_{j, k}$ denotes the contribution of the $k$ th variable to the variable of forecast error 
of the element $j$. The rows of the variance decomposition matrix $\left.\boldsymbol{\theta}_{H}\right)$ is not necessarily sum to one, each entry can be standardised by the row sum as:

$$
\left(\tilde{\boldsymbol{\theta}}_{H}\right)_{j, k}=\frac{\left(\boldsymbol{\theta}_{H}\right)_{j, k}}{\boldsymbol{\Sigma}_{k=1}^{N}\left(\boldsymbol{\theta}_{H}\right)_{j, k}}
$$

where $\boldsymbol{\Sigma}_{k=1}^{N}\left(\boldsymbol{\theta}_{H}\right)_{j, k}=1$ and the sum of all elements in $\tilde{\boldsymbol{\theta}}_{H}$ is equal to $N$. The standardised $\left(\tilde{\boldsymbol{\theta}}_{H}\right)_{j, k}$ measures the pairwise connectedness from $j$ to $k$ at horizon $\mathrm{H}$, which can be aggregated to estimate the total connectedness of the system. The connectedness measure is, therefore, calculated as the share of variance in the forecasts contributed by errors other than own errors or as the ratio of the sum of the off-diagonal elements to the sum of the entire matrix [Diebold and Yilmaz, 2012].

$$
C_{H}=100 \times \frac{\boldsymbol{\Sigma}_{j \neq k}\left(\tilde{\boldsymbol{\theta}}_{H}\right)_{j, k}}{\boldsymbol{\Sigma} \tilde{\boldsymbol{\theta}}_{H}}=100 \times\left(1-\frac{\operatorname{Tr}\{\tilde{\boldsymbol{\theta}}\}}{\mathbf{\Sigma} \tilde{\boldsymbol{\theta}}_{H}}\right)
$$

where $\operatorname{Tr}\{$.$\} is the trace operator. The connectedness measure C_{H}$ is the relative contribution to the forecast variances from the other variables in the system. The spectral representation of variance decompositions is used for measuring the frequency dynamics of the connectedness. The spectral density of $\mathbf{x}_{\mathbf{t}}$ at frequency $\omega$ maybe defined as:

$$
\mathbf{S}_{\mathbf{x}}(\omega)=\sum_{h=-\infty}^{\infty} E\left(\mathbf{x}_{t} \mathbf{x}_{t-h}^{\prime}\right) e^{-i \omega h}=\boldsymbol{\Psi}\left(e^{-i \omega}\right) \boldsymbol{\Sigma} \boldsymbol{\Psi}^{\prime}\left(e^{+i \omega}\right)
$$

where $\boldsymbol{S}_{\mathbf{x}}(\omega)$ describes how the variance the $\boldsymbol{x}_{t}$ is distributed over the frequency components $\omega$. The generalised causation spectrum over frequencies $\omega \in(-\pi, \pi)$ is defined as:

$$
(f(\omega))_{j, k}=\frac{\sigma_{k k}^{-1}\left|\left(\boldsymbol{\Psi}\left(e^{-i \omega}\right) \boldsymbol{\Sigma}\right)_{j, k}\right|^{2}}{\left(\boldsymbol{\Psi}\left(e^{-i \omega}\right) \boldsymbol{\Sigma} \mathbf{\Psi}^{\prime}\left(e^{-i \omega}\right)\right)_{j, j}},
$$

where $\boldsymbol{\Psi}\left(e^{-i \omega}\right)=\sum_{h} e^{-i \omega h} \Psi_{h}$ is the Fourier transform of the impulse response $\Psi_{h}$. It is important to note that $(f(\omega))_{j, k}$ represents the portion of the spectrum of the $i$ th variable at a given frequency $\omega$ due to shocks in the $k$ th variable. The weighting function is defined as:

$$
\Gamma_{j}(\omega)=\frac{\left(\boldsymbol{\Psi}\left(e^{-i \omega}\right) \boldsymbol{\Sigma} \boldsymbol{\Psi}^{\prime}\left(e^{-i \omega}\right)\right)_{j, j}}{\frac{1}{2 \pi} \int_{-\pi}^{\pi}\left(\boldsymbol{\Psi}\left(e^{-i \omega}\right) \boldsymbol{\Sigma} \boldsymbol{\Psi}^{\prime}\left(e^{-i \omega}\right)\right)_{j, j} d \lambda},
$$

which represents the power of $j$ th variable at a given frequency. The generalised variance decom- 
positions on frequency band $d, d=(a, b): a, b \in(-\pi, \pi)$, are defined as:

$$
\left(\boldsymbol{\theta}_{d}\right)_{j, k}=\frac{1}{2 \pi} \int_{d} \Gamma_{j}(\omega)(f(\omega))_{j, k} d \omega
$$

The scaled generalised variance decomposition on the frequency band $d, d=(a, b): a, b \in$ $(-\pi, \pi), a<b$, is defined as:

$$
\left(\tilde{\boldsymbol{\theta}}_{d}\right)_{j, k}=\left(\theta_{d}\right)_{j, k} / \sum_{k}\left(\theta_{\infty}\right)_{j, k}
$$

where $\boldsymbol{\theta}_{d}$ is defined as Equation 11 and $\left(\boldsymbol{\theta}_{\infty}\right)_{j, k}=\sum_{d_{s} \in D}\left(\boldsymbol{\theta}_{d_{s}}\right)_{j, k}$. The within connectedness on the frequency band $d$ is defined as:

$$
C_{d}^{W}=100\left(1-\frac{\operatorname{Tr}\left(\tilde{\boldsymbol{\theta}}_{d}\right)}{\sum\left(\tilde{\boldsymbol{\theta}}_{d}\right)}\right)
$$

Further, the frequency connectedness on the frequency band $d$ is defined as:

$$
C_{d}^{F}=100\left(\frac{\sum \tilde{\boldsymbol{\theta}}_{d}}{\sum \tilde{\boldsymbol{\theta}}_{\infty}}-\frac{\operatorname{Tr}\left(\tilde{\boldsymbol{\theta}}_{d}\right)}{\sum\left(\tilde{\boldsymbol{\theta}}_{d}\right)}\right)=C_{d}^{W} \frac{\sum \tilde{\boldsymbol{\theta}}_{d}}{\sum \tilde{\boldsymbol{\theta}}_{\infty}} .
$$

The within connectedness measures the connectedness effect that occurs within the frequency band and is weighted by the power of the series on the given frequency band, while the frequency connectedness decomposes the overall connectedness into distinct parts.

\section{Results}

To ensure the stationarity of the volatility data required by the VAR model, we apply the ADF, PP and KPSS tests to check the stationarity of the volatility data and the results confirm the stationarity of all variables. ${ }^{3}$ In this paper, a VAR model is used with two lags including a constant to examine volatility spillovers. ${ }^{4}$ The static and time-varying results are presented in Sections 4.1 and 4.2 , respectively.

\footnotetext{
${ }^{3}$ Unit root test results are available upon request.

${ }^{4} \mathrm{~A}$ 100-day ahead forecasting horizon for variance decomposition is used in our empirical analysis. This choice of forecasting horizon is based on Baruník and Křehlík [2018], who suggest to set the forecasting horizon to be sufficiently high. In particular, the BK18 approaches may not work well if the forecasting horizon is less than 100. In addition, we also carry out robustness checks using the 10-day forecasting horizon for both DY and BK approaches, and the corresponding static and time-varying results remain quantitatively unchanged.
} 


\subsection{Static results}

The key focus of this paper is to explore evidence of volatility spillovers between Bitcoin and blockchain patent-related stocks. Hence, after completing multiple robustness checks and comparative analyses, the net spillover of Bitcoin is identified as a more accurate measure of volatility transmission for our specific research questions. The net volatility spillover shows how much each market contributes to the volatility in other market in net terms. As we are not interested in exploring the dynamics of volatility spillovers across the fourteen stocks analysed, the net volatility spillover for each company is not reported. ${ }^{5}$ The net volatility spillover for Bitcoin is reported in Table 2, which is defined as the volatility shocks transmitted from Bitcoin to blockchain patent-related stocks minus the volatility shocks received by Bitcoin from all the stocks.

The net volatility spillover for Bitcoin is $-0.187 \%$ based on the DY12 approach and this number is further decomposed into $-0.052 \%,-0.088 \%$ and $-0.047 \%$ at three different frequencies using the BK18 approach. In other words, the gross volatility shocks transmitted from Bitcoin to blockchain patentrelated stocks is smaller than those received from blockchain related stocks, indicating volatility spillovers from the share prices of blockchain-related companies to Bitcoin play a dominant role. Based on the time-domain approach of DY12 and frequency-dependent approach of BK18, we find that blockchain patent holding companies influence Bitcoin volatility in general. Therefore, Bitcoin is a volatility receiver rather than a transmitter when all the companies are considered together. The net volatility spillover for Bitcoin tells us the result is a net one by taking into account the impacts from and towards all the companies rather than the individual net volatility spillover associated with only one company. However, it is not necessarily the case that the spillover from one company to Bitcoin is always stronger than the other way around.

\section{Insert Table 2 about here}

Next we consider the net pairwise volatility spillovers obtained from the DY12 approach for each company in Table 3. We report the net pairwise spillover as the effect of volatility shocks transmitted by Bitcoin to each blockchain patent-related company minus the volatility shocks received by Bitcoin from each company. For example, the pairwise spillover is $-0.056 \%$ for Bitcoin-Microsoft. The sum of the individual pairwise spillovers is equal to the net volatility spillover for Bitcoin $(-0.187 \%){ }^{6}$ As can be seen, the pairwise spillover is negative for all of the companies except Walmart, British Telecom and Ping An. A negative pairwise spillover would indicate that volatility shocks received by Bitcoin from a blockchain patent-related company dominate in the volatility transmission process. In other words, the volatility shocks received by Bitcoin from a company have a stronger impact

\footnotetext{
${ }^{5}$ The same applies to total and directional spillover indexes.

${ }^{6}$ This is a useful check to ensure you would interpret results correctly.
} 
than those transmitted from Bitcoin to that company. Similarly, a positive pairwise spillover would indicate that a volatility shock transmitted from Bitcoin to a blockchain patent related company was larger than those the other way around. For example, volatility shocks from Bitcoin to Walmart, British Telecom and Ping An are larger than the reverse volatility shocks given a positive pairwise spillover. The message here is that Bitcoin substantively influences the performance of Walmart, British Telecom and Ping An.

Further, we calculate the ratio of negative pairwise spillovers to the sum of negative pairwise spillovers to see the proportion of contributions of each company to the volatility spillover to Bitcoin in lower panel of Table 3. As the pairwise spillovers for Walmart, British Telecom and Ping An are positive, they are excluded in this calculation. We identify the top three companies contributing to the volatility spillover to Bitcoin as: Microsoft (0.254 or 25.364\%), Mastercard (22.120\%) and Intel $(14.318 \%)$. The above three identified US firms contribute a total of $61.802 \%$ to the sum of negative pairwise spillovers to Bitcoin, highlighting the importance in driving Bitcoin risk. Moreover, we find that Baidu has the largest contribution to volatility spillovers $(8.17 \%)$ to Bitcoin among the Chinese firms followed by Alibaba (4.09\%), JD (2.21\%) and Tencent (1.13\%). In sum, the contributions made by the Chinese firms to the volatility spillover to Bitcoin are small. Overall, the calculated spillover effects on Bitcoin from the US companies are larger than the other companies.

\section{Insert Tables 3 and 4 about here}

We now apply the BK18 approach to decompose the net pairwise volatility spillovers to see if the results would change at different frequencies. Table 4 presents net pairwise volatility spillovers using the BK18 approach. Similarly, we also calculate the ratio of negative pairwise spillovers to the sum of negative pairwise spillovers in Table 4 at three frequencies to take into account short, medium and long term shocks.

The short term (frequency one) results are presented in the upper panel of Table 4. As can be seen, the pairwise spillovers for most companies are negative with the exception of British Telecom and Ping An. Those companies with a negative pairwise spillover would indicate that volatility shocks received by Bitcoin from a blockchain patent related company possess a higher effect vice versa, suggesting that the performance of these companies substantively influences Bitcoin risk. As we can see from the calculated spillovers to Bitcoin, the proportion of contributions made by each company to the volatility spillover to Bitcoin are different. The top 3 companies contributing the volatility spillover to Bitcoin are Visa (0.1750 or 17.50\%), Microsoft (14.66\%) and Mastercard $(13.97 \%)$. In addition, Accenture is the fourth ranked contributor with a spillover effect of $12.32 \%$. Baidu contributes approximately $7.12 \%$ of the calculated spillovers to Bitcoin. The spillover effect to Bitcoin from Baidu is similar to those of IBM (8.09\%) and BofA (7.72\%).

The medium term effects (frequency two) are shown as the middle panel of the table. The 
pairwise spillovers are negative for the majority companies except Walmart, British Telecom and Ping An. The calculated spillovers to Bitcoin provide us information about the contributions from each company to Bitcoin between 5 and 10 days. Microsoft (25.08\%), Mastercard (21.50\%) and Intel $(12.65 \%)$ are found to play a more important role in driving up the volatility of Bitcoin. Moreover, we notice that the calculated spillover effects from Accenture towards Bitcoin decrease sharply from $12.32 \%$ in the short term to $4.40 \%$ in the medium term. Compared with results in Table 3, the calculated spillovers from Accenture (4.851\%) based on the DY12 approach is mainly driven by the medium term spillovers of the BK18 approach. Similarly, the calculated spillovers for IBM also drop sharply from $8.09 \%$ in the short term to $1.94 \%$ in the medium term.

In the long term (frequency three), the pairwise spillovers are negative except IBM, Walmart, Accenture, Ping An and Tencent. At the longer horizon, we observe that Bitcoin dominates in the volatility transmission process towards IBM, Walmart, Accenture, Ping An and Tencent while the remainder of the blockchain companies dominate in the volatility transmission process towards Bitcoin. Of particular interest is that the pairwise spillovers for IBM, Accenture and Tencent are becoming positive at the frequency three only. At frequency three, the calculated spillovers to Bitcoin provide us information about the contributions from each company to Bitcoin in the long term ( $>10$ days). The top 3 contributors remain the same as those of companies at the frequency two including Microsoft (30.62\%), Mastercard (26.21\%) and Intel (21.04\%). These three companies together contribute to a combination spillover of $77.87 \%$ towards Bitcoin at frequency three compared with the counterpart of $59.23 \%$ at frequency two, indicating that these three leading blockchain patent holders have more influences on Bitcoin risk especially in the long term.

In general, the share prices of US-based Microsoft, Mastercard, Intel and Visa play a critical role in contributing to the volatility of Bitcoin. These results are, in general, consistent with those reported in Table 3 using the DY12 approach. Of particular interest is that the frequency-domain approach of BK18 offers more insights in measuring the connectedness at different frequencies. In addition to the above, there are several interesting findings. When we look at the calculated spillovers to Bitcoin across three frequencies, the calculated spillover effects to Bitcoin from Intel, Microsoft and Mastercard increase from short- to long-term. On the other hand, the calculated spillover effects towards Bitcoin for BofA and Visa decrease from the short- to long-term. Ping An remains the only company with positive pairwise spillovers across three different frequencies, indicating that Bitcoin is a volatility transmitter for Ping An. BT has a positive pairwise spillover in the short- and medium term while Walmart has a positive pairwise spillover in the medium and long-term only.

\subsection{Time-varying results}

We use the rolling window approach to capture the time-varying features of net spillovers and

pairwise spillovers from blockchain-patent companies to Bitcoin over a two hundred day period at 
the time of each announcement as presented in Figure 3. As observed, the net spillovers for Bitcoin remain negative for the majority of the time. There are substantial changes in the estimates of the net spillovers for Bitcoin across the sample period and they are highly volatile based on the two approaches. The time-varying net spillovers for Bitcoin based on the DY12 approach are shown as Figure 3a and the corresponding estimates based on BK18 approach at short-, medium and long-term are displayed as Figures $3 \mathrm{~b}$ to $3 \mathrm{~d}$, respectively. When we look at time-varying DY12 net spillovers for Bitcoin in Figure 3a, the net spillovers reach the largest magnitude in early 2018. Such a pattern is also observed in Figure 3d using the BK approach for the long term (frequency 3). Overall, the time-varying based evidence favours volatility spillovers transmitted from the blockchain patent-related share prices to the Bitcoin market, which are dominant in the volatility transmission process. Table 5 also shows the maxima, minima, means and standard deviations of the spillover estimates. These estimates are also consistent with the movements of the time-varying spillover effects in Figure 3.

\section{Insert Figure 3 about here}

We further analyse which company has the largest recorded volatility transmission to Bitcoin by focusing on the time-varying pairwise spillovers. The maxima, minima, means and standard deviations of the pairwise spillovers estimates are presented in Tables 6 and 7 based on both approaches. In particular for the estimates based on the BK18 approach, moving from high frequency (frequency one) to low frequency (frequency three), the mean pairwise spillovers of twelve of fourteen companies monotonically increase in size except Walmart and Ping An.

\section{Insert Tables 6 through 7 about here}

Figure 4 presents the time-varying pairwise spillovers results for DY12, where there are three interesting empirical findings. First, the rolling window based estimates show time-varying spillover effects for each company. Second, the pairwise volatility spillovers are negative for most of the time except British Telecom and Ping An. During the sample period in 2017-18, the pairwise spillovers between Bitcoin and Ping An increased sharply in size and remained negative for most of the time. After early 2018, the pairwise spillovers became positive, indicating that Bitcoin had more influences on the volatility transmission towards Ping An. For BT, the corresponding estimates show some different results, where its pairwise spillovers remain positive from 2017 until the mid 2018. This result suggests that the volatility spillovers from Bitcoin to BT are stronger than the opposite direction. Third, the volatility spillovers from Intel, Mastercard, Visa and Microsoft to Bitcoin increased dramatically in size from 2017 to early 2018, corresponding to a dramatic rise and sharp fall of Bitcoin prices during this period. The rolling window based DY12 evidence provides 
more insights into the connectedness in contrast with those of the static DY12 results reported in Section 4.1.

\section{Insert Figure 4 about here}

Figures 5 to 6 illustrate results based on BK18 for the short-, medium- and long-term respectively. Firstly, the movements of the time-varying pairwise volatility spillovers exhibit oscillations below zero across most of the sample period for all the companies except British Telecom, Ping An and Tencent. This result is evident across all three time frequencies analysed.

\section{Insert Figures 5 to 6 about here}

Secondly, in the short term, Bitcoin shocks influence the risk levels of Mastercard, Visa and Alibaba as the corresponding pairwise spillovers are positive during the development phase of blockchain patent application between mid-2016 and early 2017. Thirdly, in both the mediumand long-term, we observe that the pairwise volatility spillovers from at least eight out of the fourteen blockchain-patent-related companies to Bitcoin have increased in size during the period from mid-2017 to early 2018. These eight companies include IBM, Intel, BofA, Mastercard, Visa, Microsoft, JD and Alibaba. This is particularly evident when comparing the pairwise volatility spillovers on the $y$-axis from frequency one (1-4 days) through frequency three ( $>10$ days). This particular period of time is often described (Corbet et al. [2018]) as the 'Bitcoin bubble', where the Bitcoin price reached its all time high of approximately US\$20,000 in December 2017. Our results show that, at that time, the long-term shocks from these companies had a larger effect on Bitcoin volatility than the short-term counterparts.

Finally, during the Bitcoin bubble, we observe that there was a sharp negative spike, of around $-0.3 \%$, of pairwise volatility spillovers at frequency three to Bitcoin from four companies: Intel, Mas-

tercard, Visa and Microsoft. These results suggest that announcements from these four companies played a critical role in driving the volatility of Bitcoin.

\section{Conclusion}

Our research sheds light on the connectedness between blockchain patent related stock prices and Bitcoin prices using the time-domain and frequency-domain approaches of Diebold and Yilmaz [2012] and Baruník and Křehlík [2018]. We present both static and time-varying results in this paper, where the rolling window based results, in particular, allow us to provide more insights into the connectedness in contrast to those based on the static formulation. Specifically, we find that 
volatility spillovers from blockchain patent related stock prices to Bitcoin are more intensive than that calculated in the opposite direction. Among the blockchain patent holders, our results suggest that several companies play a critical role influencing the volatility of Bitcoin, especially for the period between mid-2017 to early 2018. Not all, the blockchain patent-related stock prices contribute equally to Bitcoin during that period, but these results suggest an avenue through which large corporation can influence cryptocurrency prices through their announcements of future technological intentions. Among the blockchain patent holders, our results suggest that several companies played a substantial role when adding to the already substantial volatility of Bitcoin markets, especially for the period from mid-2007 throughout early 2018. Evidence suggests that not all the blockchain patent-related companies analysed contribute equally to Bitcoin during that period. However, the decision of a number of very large companies as ranked by market capitalisation appear to have had a re-affirming effect on cryptocurrency traders, with positive signals generated in the decision for these companies to enter this developing markets. However, the inherent risks incorporated with blockchain and cryptocurrency patent-development appears to have been passed to unsuspecting investors in some cases as contagion effects increase. Such contagion merits further investigation as particular warnings should perhaps not only be presented to those companies with no evidence of prior exposure and market knowledge, but also to the investors who provide their financing of the true risks that they are now exposed through such directions of technological development.

The findings in this paper have important policy implications. Firstly, our results clearly suggest that the performance of FinTech companies have a strong impact on the risk level of the Bitcoin market. For regulatory authorities, special attention may need to be paid to the blockchain related companies as they substantially contribute to the volatility of Bitcoin. Secondly, we show for the first time in the literature that the performance of several patent development companies contributes to the all-time high for Bitcoin in the period 2017 through 2018. Overall, the findings presented here contribute to our understanding of the pricing of Bitcoin and hence volatility of Bitcoin. However, when considering the breadth of research that continues to identify issues surrounding exceptional levels of volatility, contagion and potential irrational exuberance, such corporate entities that partake in FinTech and cryptocurrency patent development must take care to assess these new risks that they are incorporating into their corporate structure.

\section{References}

Aharon, D. and M. Qadan (2019). Bitcoin and the day-of-the-week effect. Finance Research Letters 31, 415-424.

Ahluwalia, S., R. Mahto, and M. Guerrero (2020). Blockchain technology and startup financing: A transaction cost economics perspective. Technological Forecasting and Social Change 151.

Akhtaruzzaman, M., A. Sensoy, and S. Corbet (2019). The influence of bitcoin on portfolio diversification and design. Finance Research Letters. 
Akyildirim, E., S. Corbet, B. Lucey, A. Sensoy, and L. Yarovaya (2019). The relationship between implied volatility and cryptocurrency returns. Finance Research Letters.

Alexander, C. and M. Dakos (2020). A critical investigation of cryptocurrency data and analysis. Quantitative Finance 20(2), 173-188.

Alvarez-Ramirez, J., E. Rodriguez, and C. Ibarra-Valdez (2018). Long-range correlations and asymmetry in the bitcoin market. Physica A: Statistical Mechanics and its Applications 492, 948-955.

Ammous, S. (2018). Can cryptocurrencies fulfil the functions of money? Quarterly Review of Economics and Finance 70, 38-51.

Antonakakis, N., I. Chatziantoniou, and D. Gabauer (2019). Cryptocurrency market contagion: Market uncertainty, market complexity, and dynamic portfolios. Journal of International Financial Markets, Institutions and Money 61, 37-51.

Balcilar, M., E. Bouri, R. Gupta, and D. Roubaud (2017). Can volume predict bitcoin returns and volatility? a quantiles-based approach. Economic Modelling 64, 74-81.

Bartram, S., G. Brown, and R. Stulz (2012). Why are u.s. stocks more volatile? Journal of Finance 67(4), 1329-1370.

Baruník, J. and T. Křehlík (2018). Measuring the frequency dynamics of financial connectedness and systemic risk. Journal of Financial Econometrics 16(2), 271-296.

Baur, D., K. Hong, and A. Lee (2018). Bitcoin: Medium of exchange or speculative assets? Journal of International Financial Markets, Institutions and Money 54, 177-189.

Bohme, R., N. Christin, B. Edelman, and T. Moore (2015). Bitcoin: Economics, technology, and governance. Journal of Economic Perspectives 29(2), 213-238.

Brandvold, M., P. Molnár, K. Vagstad, and O. Andreas Valstad (2015). Price discovery on bitcoin exchanges. Journal of International Financial Markets, Institutions and Money 36, 18-35.

Briere, M., K. Oosterlinck, and A. Szafarz (2015). Virtual currency, tangible return: Portfolio diversification with bitcoin. Journal of Asset Management 16(6), 365-373.

Cagli, E. (2019). Explosive behavior in the prices of bitcoin and altcoins. Finance Research Letters 29, 398-403.

Cahill, D., D. G. Baur, Z. (Frank) Liu, and J. W. Yang (2020). I am a blockchain too: How does the market respond to companies' interest in blockchain? Journal of Banking and Finance 113.

Celeste, V., S. Corbet, and C. Gurdgiev (2019). Fractal dynamics and wavelet analysis: Deep volatility and return properties of bitcoin, ethereum and ripple. Quarterly Review of Economics and Finance.

Chaim, P. and M. Laurini (2019). Is bitcoin a bubble? Physica A: Statistical Mechanics and its Applications 517, $222-232$.

Cheah, E.-T. and J. Fry (2015). Speculative bubbles in bitcoin markets? an empirical investigation into the fundamental value of bitcoin. Economics Letters 130, 32-36.

Chen, M., Q. Wu, and B. Yang (2019). How valuable is fintech innovation? Review of Financial Studies 32(5), 2062-2106. 
Cheng, S., G. De Franco, H. Jiang, and P. Lin (2019). Riding the blockchain mania: Public firms' speculative 8-k disclosures. Management Science 65(12), 5901-5913.

Cheung, A.-K., E. Roca, and J.-J. Su (2015). Crypto-currency bubbles: an application of the phillips-shi-yu (2013) methodology on mt. gox bitcoin prices. Applied Economics 47(23), 2348-2358.

Chow, W. and M. Fung (2008). Volatility of stock price as predicted by patent data: An mgarch perspective. Journal of Empirical Finance 15(1), 64-79.

Chu, J., S. Chan, and Y. Zhang (2020). High frequency momentum trading with cryptocurrencies. Research in International Business and Finance 52.

Ciaian, P., M. Rajcaniova, and D. Kancs (2018). Virtual relationships: Short- and long-run evidence from bitcoin and altcoin markets. Journal of International Financial Markets, Institutions and Money 52, 173-195.

Corbet, S., C. Larkin, B. Lucey, A. Meegan, and L. Yarovaya (2020). Cryptocurrency reaction to fomc announcements: Evidence of heterogeneity based on blockchain stack position. Journal of Financial Stability 46.

Corbet, S., C. Larkin, B. Lucey, and L. Yarovaya (2019). Kodakcoin: a blockchain revolution or exploiting a potential cryptocurrency bubble? Applied Economics Letters.

Corbet, S., B. Lucey, M. Peat, and S. Vigne (2018). Bitcoin futures - what use are they? Economics Letters 172, $23-27$.

Corbet, S., B. Lucey, M. Peat, and S. Vigne (2019). What sort of asset? bitcoin analysed. Lecture Notes in Business Information Processing 345, 52-65.

Corbet, S., B. Lucey, A. Urquhart, and L. Yarovaya (2019). Cryptocurrencies as a financial asset: A systematic analysis. International Review of Financial Analysis 62, 182-199.

Corbet, S., B. Lucey, and L. Yarovaya (2018). Datestamping the bitcoin and ethereum bubbles. Finance Research Letters 26, 81-88.

Corbet, S., A. Meegan, C. Larkin, B. Lucey, and L. Yarovaya (2018a). Exploring the dynamic relationships between cryptocurrencies and other financial assets. Economics Letters 165, 28-34.

Corbet, S., A. Meegan, C. Larkin, B. Lucey, and L. Yarovaya (2018b). Exploring the dynamic relationships between cryptocurrencies and other financial assets. Economics Letters 165, 28-34.

Cretarola, A. and G. Figà-Talamanca (2019). Detecting bubbles in bitcoin price dynamics via market exuberance. Annals of Operations Research.

Das, D. and A. Dutta (2020). Bitcoin's energy consumption: Is it the achilles heel to miner's revenue? Economics Letters 186.

Diebold, F. X. and K. Yilmaz (2012). Better to give than to receive: Predictive directional measurement of volatility spillovers. International Journal of Forecasting 28(1), 57-66.

Dwyer, G. (2015). The economics of bitcoin and similar private digital currencies. Journal of Financial Stability 17, 81-91.

Eross, A., F. McGroarty, A. Urquhart, and S. Wolfe (2019). The intraday dynamics of bitcoin. Research in International Business and Finance 49, 71-81. 
Fisch, C. (2019). Initial coin offerings (icos) to finance new ventures. Journal of Business Venturing 34(1), 1-22.

Foley, S., J. Karlsen, and T. Putnins (2019). Sex, drugs, and bitcoin: How much illegal activity is financed through cryptocurrencies? Review of Financial Studies 32(5), 1798-1853.

Gandal, N., J. Hamrick, T. Moore, and T. Oberman (2018). Price manipulation in the bitcoin ecosystem. Journal of Monetary Economics 95, 86-96.

Geuder, J., H. Kinateder, and N. Wagner (2019). Cryptocurrencies as financial bubbles: The case of bitcoin. Finance Research Letters 31, 179-184.

Hu, Y., H. Valera, and L. Oxley (2019). Market efficiency of the top market-cap cryptocurrencies: Further evidence from a panel framework. Finance Research Letters 31, 138-145.

Ibikunle, G., F. McGroarty, and K. Rzayev (2020). More heat than light: Investor attention and bitcoin price discovery. International Review of Financial Analysis 69.

Ji, Q., E. Bouri, C. Lau, and D. Roubaud (2019). Dynamic connectedness and integration in cryptocurrency markets. International Review of Financial Analysis 63, 257-272.

Jin, J., J. Yu, Y. Hu, and Y. Shang (2019). Which one is more informative in determining price movements of hedging assets? evidence from bitcoin, gold and crude oil markets. Physica A: Statistical Mechanics and its Applications 527.

Katsiampa, P. (2017). Volatility estimation for bitcoin: A comparison of garch models. Economics Letters 158, 3-6.

Katsiampa, P., S. Corbet, and B. Lucey (2019). High frequency volatility co-movements in cryptocurrency markets. Journal of International Financial Markets, Institutions and Money 62, 35-52.

Koutmos, D. (2018). Return and volatility spillovers among cryptocurrencies. Economics Letters 173, 122-127.

Kristoufek, L. (2019). Is the bitcoin price dynamics economically reasonable? evidence from fundamental laws. Physica A: Statistical Mechanics and its Applications 536.

Lin, S. (2015). Are ivory towers truly ivory? knowledge spillovers and firm innovation. Journal of Economics and Business 80, 21-36.

Matkovskyy, R. (2019). Centralized and decentralized bitcoin markets: Euro vs usd vs gbp. Quarterly Review of Economics and Finance 71, 270-279.

Meegan, A., S. Corbet, and C. Larkin (2018). Financial market spillovers during the quantitative easing programmes of the global financial crisis (2007-2009) and the european debt crisis. Journal of International Financial Markets, Institutions and Money 56, 128-148.

Pan, X., X. Chen, and L. Ning (2018). Exploitative technological diversification, environmental contexts, and firm performance. Management Decision 56(7), 1613-1629.

Pesaran, H. H. and Y. Shin (1998). Generalized impulse response analysis in linear multivariate models. Economics letters 58(1), 17-29.

Symitsi, E. and K. J. Chalvatzis (2018). Return, volatility and shock spillovers of bitcoin with energy and technology companies. Economics Letters 170, 127-130. 
Vitt, C. and H. Xiong (2016). The impact of patent activities on stock dynamics in the high-tech sector. Volume 2016-January, pp. 399-408.

Wei, W. (2018a). The impact of tether grants on bitcoin. Economics Letters 171, 19-22.

Wei, W. (2018b). Liquidity and market efficiency in cryptocurrencies. Economics Letters 168, 21-24.

Xiong, J., Q. Liu, and L. Zhao (2019). A new method to verify bitcoin bubbles: Based on the production cost. North American Journal of Economics and Finance.

Yi, S., Z. Xu, and G.-J. Wang (2018). Volatility connectedness in the cryptocurrency market: Is Bitcoin a dominant cryptocurrency? International Review of Financial Analysis 60, 98-114.

Zeng, S., X. Liu, X. Li, Q. Wei, and Y. Shang (2019). Information dominance among hedging assets: Evidence from return and volatility directional spillovers in time and frequency domains. Physica A: Statistical Mechanics and its Applications 536.

Zhang, Y., S. Chan, J. Chu, and S. Nadarajah (2019). Stylised facts for high frequency cryptocurrency data. Physica A: Statistical Mechanics and its Applications 513, 598-612. 
Figure 1: Number of blockchain patent applications worldwide (as of April 2019)

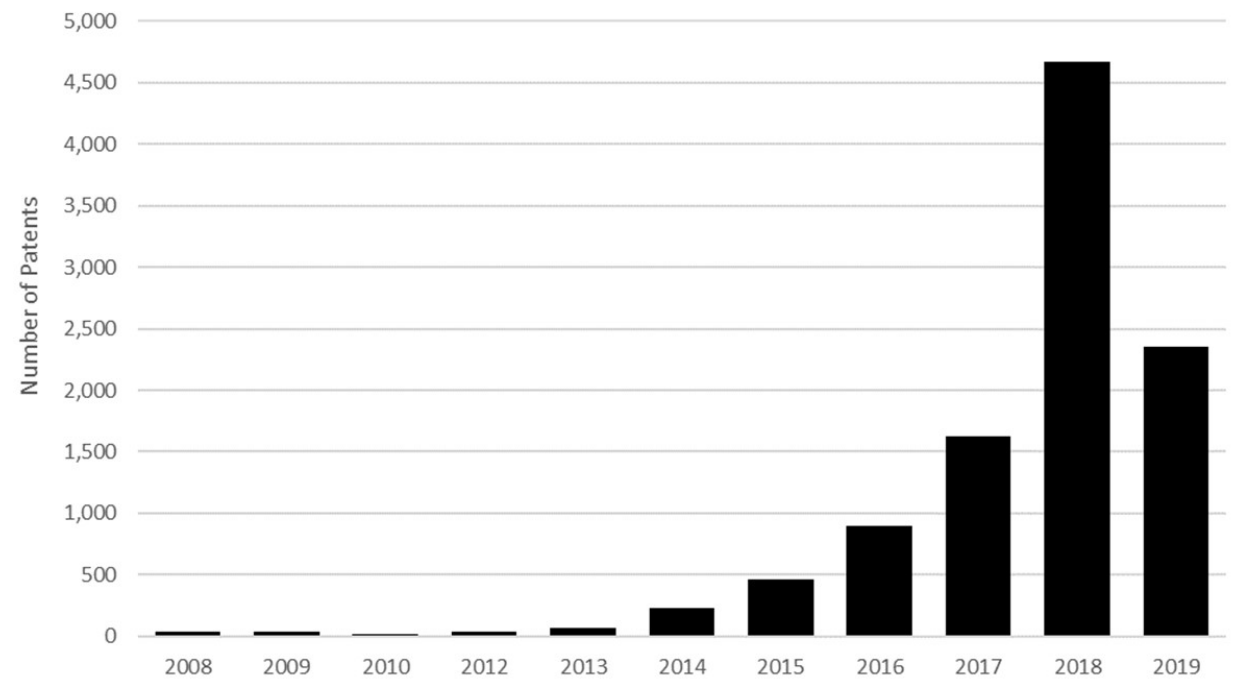

Note: The above figure is based on data obtained from https://www.statista.com/ 
Figure 2: Time series plot of volatility data

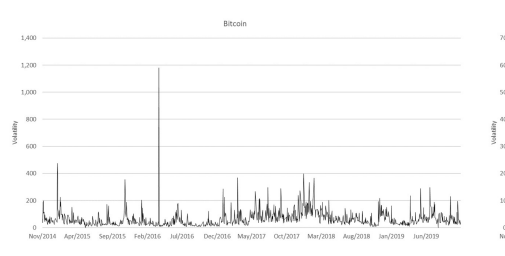

wesereses

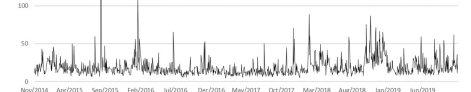
sme

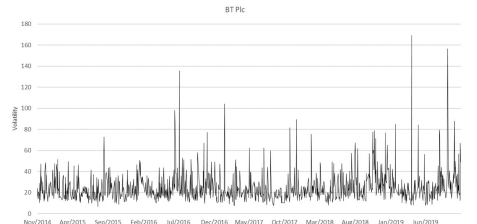

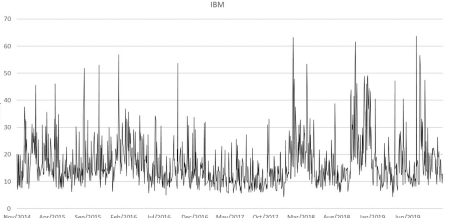
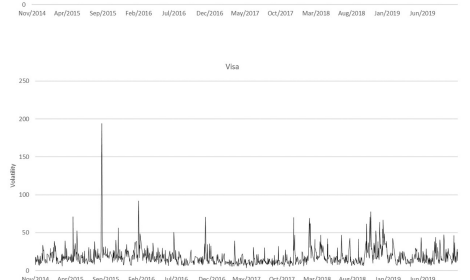
stement

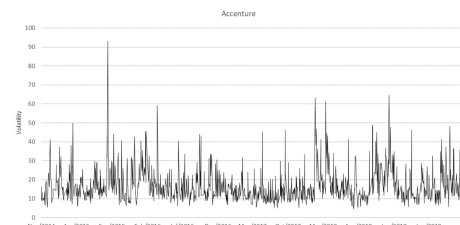

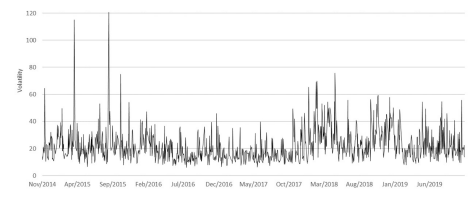

mosoest
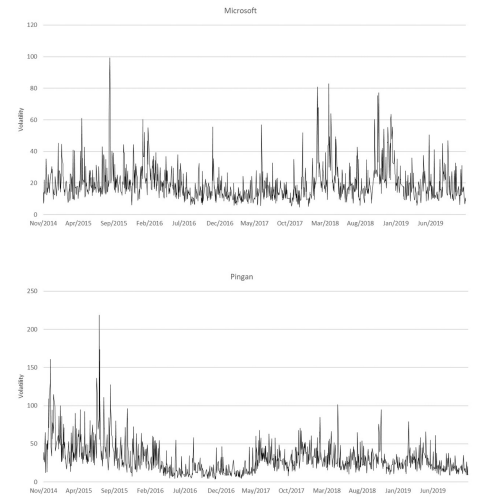

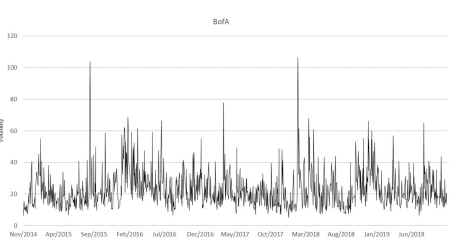

wames
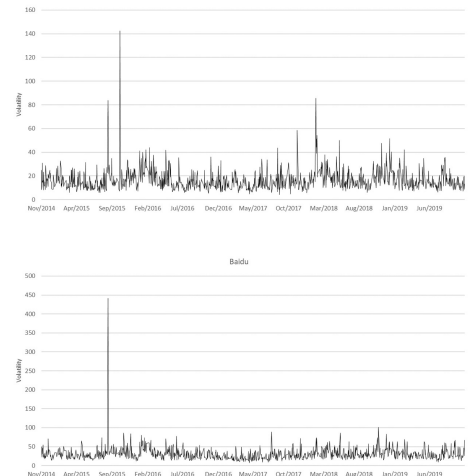

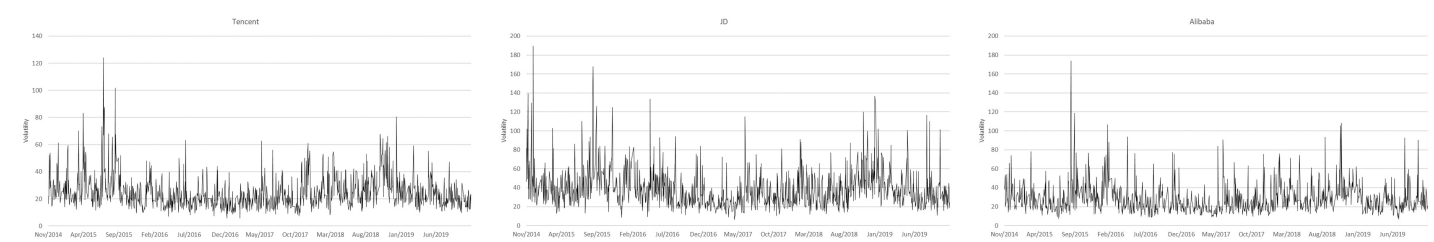

Note: The above figure presents the data for the period between 10 November 2014 through 07 November 2019 
Figure 3: Rolling window estimates for net spillovers of Bitcoin using the Diebold and Yilmaz [2012] and Baruník and Křehlík [2018] methodologies
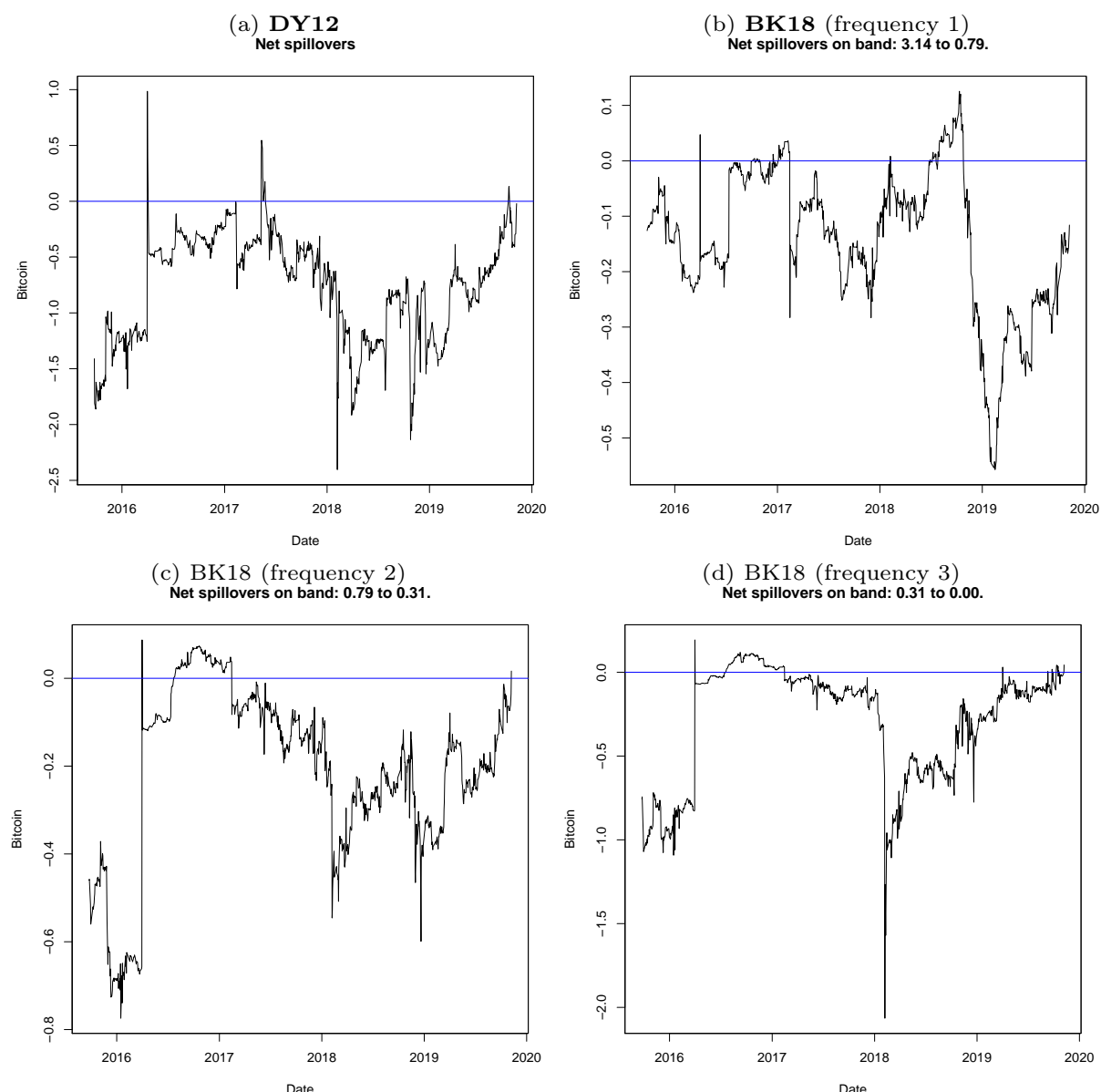

Note: The above figure presents the rolling window estimates for spillovers between Bitcoin (BTC) and the stated blockchain patent-related stocks across multiple time frequencies using the approach developed by both Diebold and Yilmaz [2012] (DY12) and Baruník and Křehlík [2018] (BK18). 
Figure 4: Rolling window estimates for pairwise spillovers using the Diebold and Yilmaz [2012] approach.
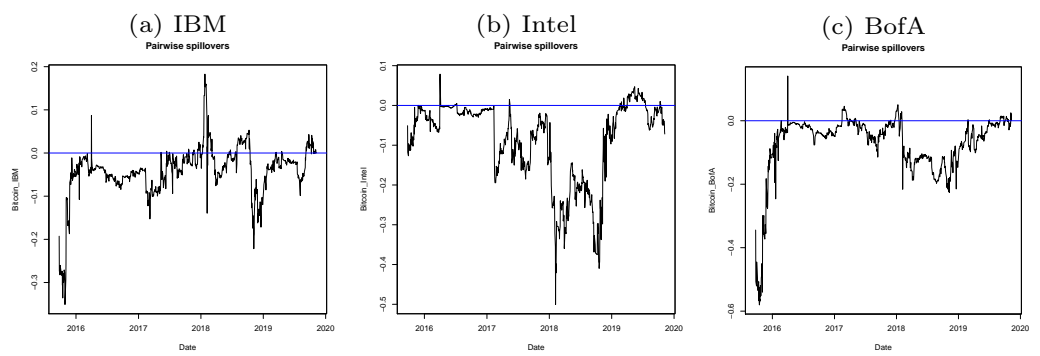

(d) Mastercard

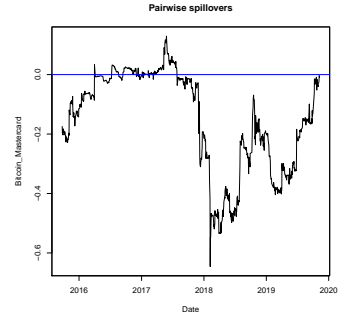

(i) Accenture

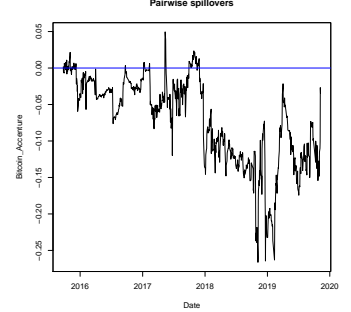

$\underset{\text { Pairuise spillowers }}{\text { (e) Visa }}$

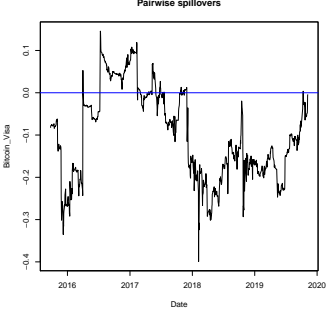

(j) Ping An

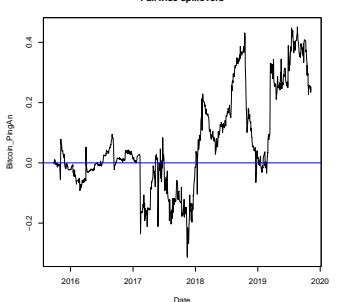

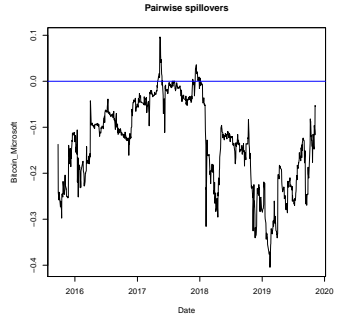
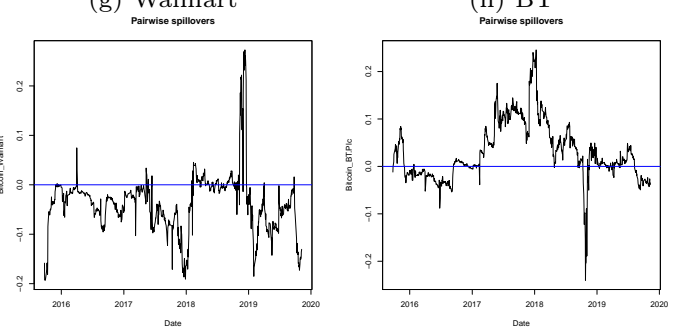

(m) JD

(n) Alibaba
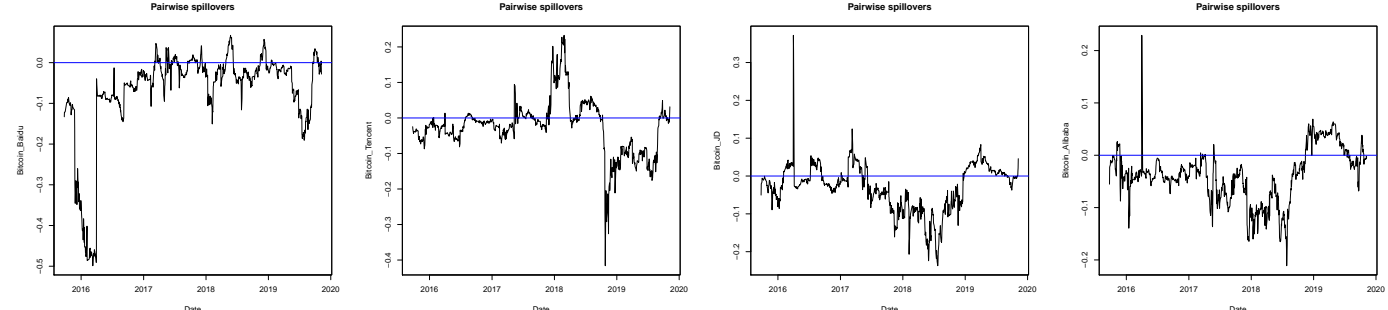

Note: The above figure presents the rolling window estimates for pairwise spillovers between Bitcoin (BTC) and the stated blockchain patent-related stocks across multiple time frequencies using the approach developed by Diebold and Yilmaz [2012] (DY12). 
Figure 5: Rolling window estimates for pairwise spillovers using the Baruník and Křehlík [2018] approach over frequency 1 (1-4 days)
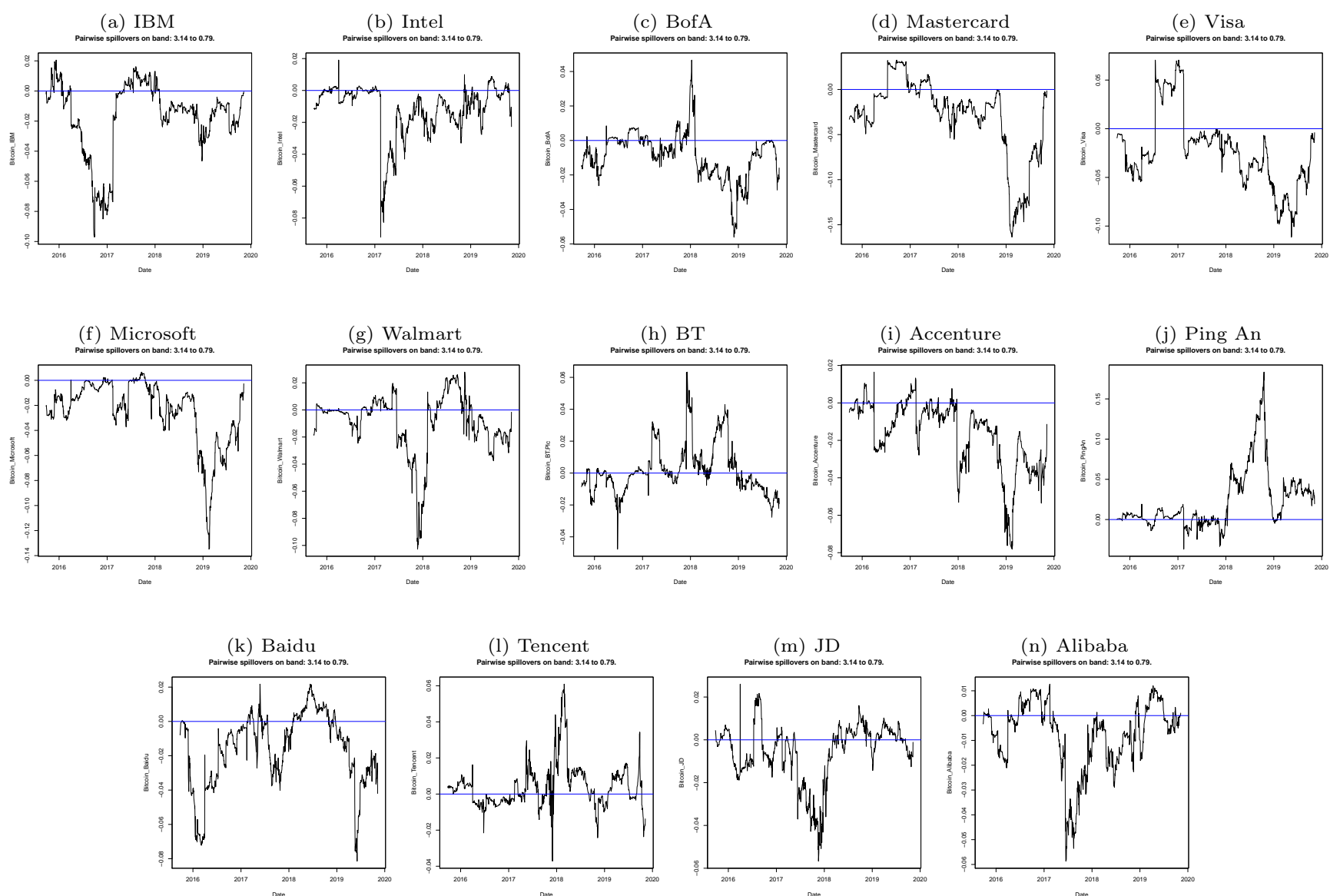

Note: The above table presents the rolling window estimates for pairwise spillovers between Bitcoin (BTC) and the stated blockchain patent-related stocks across the selected first frequency (1-4 days) using the approach developed by Baruník and Křehlík [2018] (BK18). 
Figure 6: Rolling window estimates for pairwise spillovers using the Baruník and Křehlík [2018] approach over frequency 2 (5-10 days)
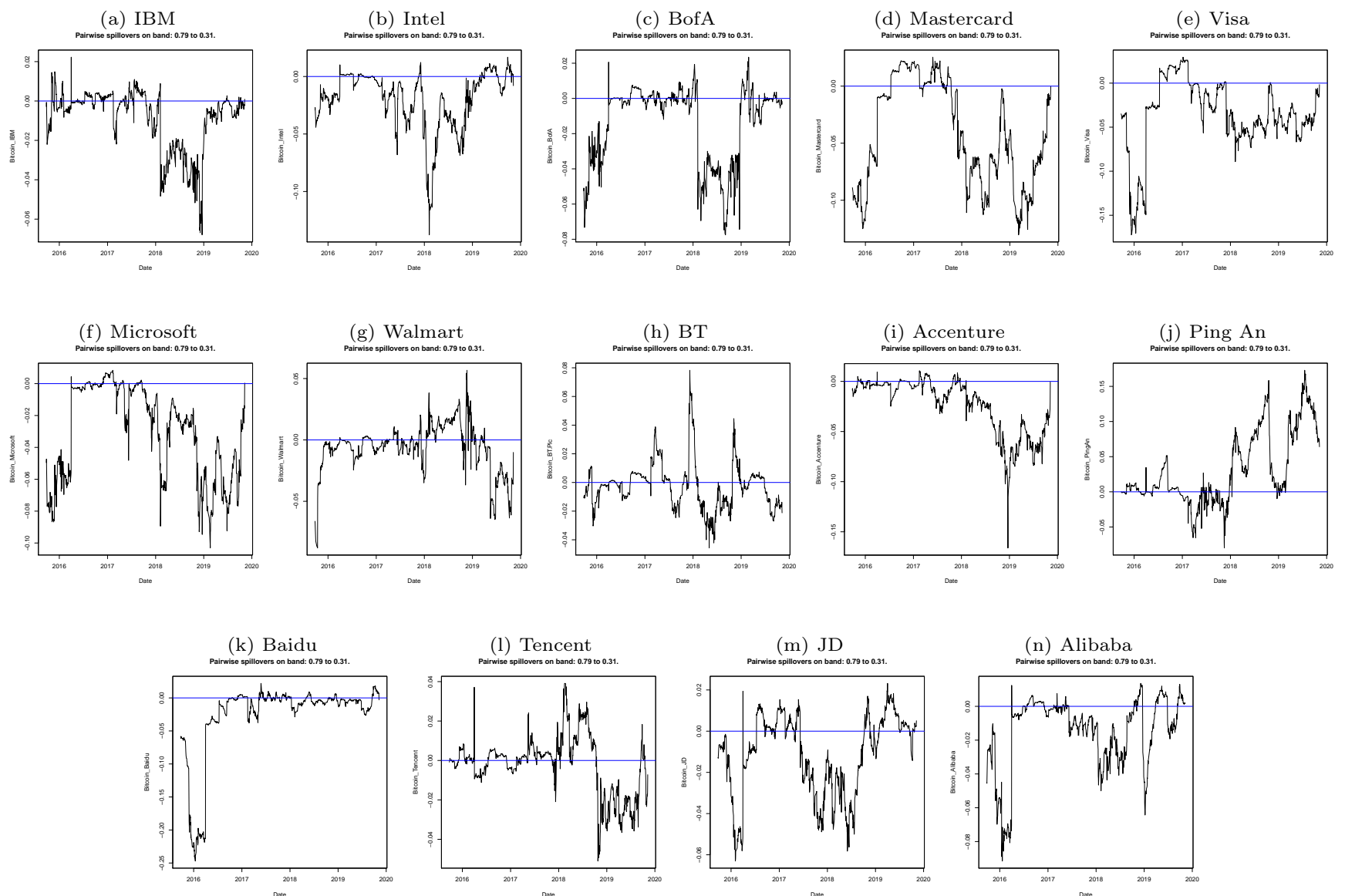

Note: The above table presents the rolling window estimates for pairwise spillovers between Bitcoin (BTC) and the stated blockchain patent-related stocks across the selected second frequency (5-10 days) using the approach developed by Baruník and Křehlík [2018] (BK18). 
Figure 7: Rolling window estimates for pairwise spillovers using the Baruník and Křehlík [2018] approach over frequency 3 (>10 days)
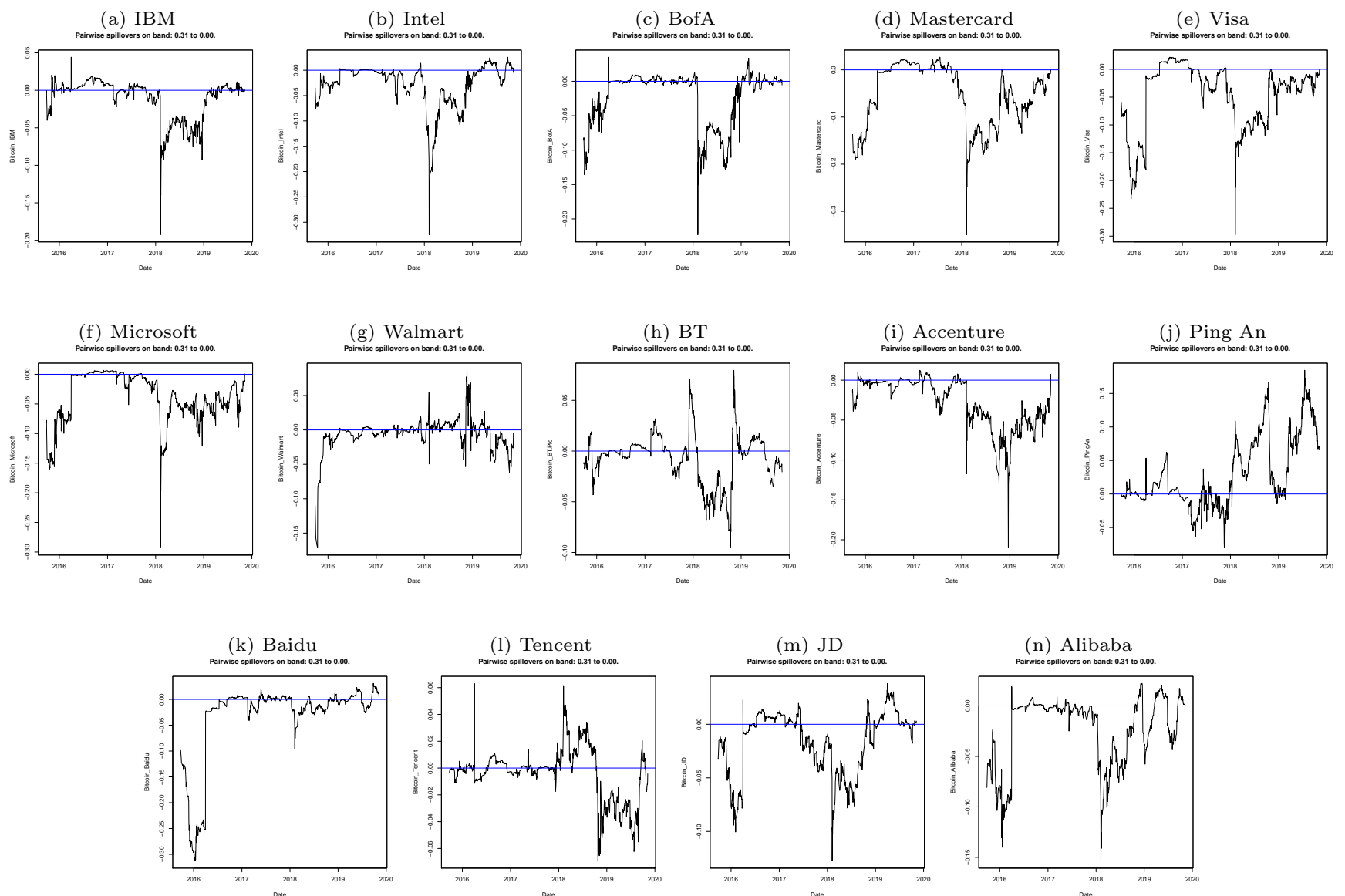

Note: The above table presents the rolling window estimates for pairwise spillovers between Bitcoin (BTC) and the stated blockchain patent-related stocks across the selected third frequency (>10 days) using the approach developed by Baruník and Křehlík [2018] (BK18). 
Table 1: Descriptive statistics of the volatility data.

\begin{tabular}{|c|c|c|c|c|c|}
\hline & BTC & IBM & Intel & BofA & Mastercard \\
\hline Mean & 66.0938 & 16.5404 & 21.8621 & 22.5808 & 19.2077 \\
\hline Median & 48.6289 & 14.1721 & 18.9739 & 19.75949 & 15.8361 \\
\hline $\operatorname{Max}$ & 1181.4870 & 63.6680 & 120.8037 & 106.3586 & 222.2631 \\
\hline Min & 0.0000 & 4.2794 & 6.1265 & 4.9916 & 4.7018 \\
\hline \multirow[t]{2}{*}{ Std.Dev. } & 64.67318 & 8.7295 & 11.3210 & 11.5251 & 12.8424 \\
\hline & Visa & Microsoft & Walmart & $\mathrm{BT}$ & Accenture \\
\hline Mean & 17.9414 & 19.5560 & 16.5041 & 24.6974 & 16.6956 \\
\hline Median & 15.0480 & 16.8037 & 14.4016 & 21.3067 & 14.3884 \\
\hline $\operatorname{Max}$ & 194.2000 & 99.3254 & 142.4465 & 169.1068 & 93.08655 \\
\hline Min & 3.8148 & 4.7353 & 4.2485 & 6.6745 & 4.6148 \\
\hline \multirow[t]{2}{*}{ Std.Dev. } & 11.5879 & 10.9960 & 8.9060 & 14.0959 & 8.8279 \\
\hline & Ping An & Baidu & Tencent & JD & Alibaba \\
\hline Mean & 30.1852 & 30.6696 & 24.7598 & 38.8952 & 28.5799 \\
\hline Median & 25.2857 & 27.2316 & 21.8294 & 34.9638 & 24.8271 \\
\hline $\operatorname{Max}$ & 218.6643 & 441.8481 & 124.2227 & 189.5500 & 173.7770 \\
\hline Min & 3.6123 & 7.0749 & 5.6472 & 6.2359 & 6.6230 \\
\hline Std.Dev. & 20.7851 & 18.3469 & 12.1954 & 20.0459 & 15.2220 \\
\hline
\end{tabular}

Table 2: Net volatility spillovers of Bitcoin.

\begin{tabular}{c|ccc}
\hline \hline \multicolumn{4}{c}{ Net volatility spillovers of Bitcoin in \% } \\
\hline DY12 & \multicolumn{3}{c}{ BK18 } \\
& $\begin{array}{c}\text { Freq. } \mathbf{1} \\
(1-4 \text { days })\end{array}$ & $\begin{array}{c}\text { Freq. } \mathbf{2} \\
(5-10 \text { days })\end{array}$ & $\begin{array}{c}\text { Freq. } \mathbf{3} \\
(>10 \text { days })\end{array}$ \\
\hline-0.1873 & -0.0524 & -0.0879 & -0.0469 \\
\hline \hline
\end{tabular}

Note: The above table presents the rolling window estimates for volatility spillovers from Bitcoin (BTC) across multiple time frequencies using the approach developed by Diebold and Yilmaz [2012] (DY12).

Table 3: Pairwise spillovers using the approach developed by Diebold and Yilmaz [2012] (DY12)

\begin{tabular}{|c|c|c|c|c|}
\hline \multicolumn{5}{|c|}{ Pairwise spillovers using DY12 in \% } \\
\hline $\begin{array}{l}\text { BTC-IBM } \\
-0.0036\end{array}$ & $\begin{array}{l}\text { BTC-Intel } \\
-0.0318\end{array}$ & $\begin{array}{l}\text { BTC-BofA } \\
-0.0104\end{array}$ & $\begin{array}{c}\text { BTC-Mastercard } \\
-0.0491\end{array}$ & $\begin{array}{l}\text { BTC-Visa } \\
-0.0255\end{array}$ \\
\hline $\begin{array}{c}\text { BTC-Microsoft } \\
-0.0564\end{array}$ & $\begin{array}{c}\text { BTC-Walmart } \\
0.0082\end{array}$ & $\begin{array}{l}\text { BTC-BT } \\
0.0069\end{array}$ & $\begin{array}{c}\text { BTC-Accenture } \\
-0.0108\end{array}$ & $\begin{array}{c}\text { BTC-Ping An } \\
0.0198\end{array}$ \\
\hline $\begin{array}{l}\text { BTC-Baidu } \\
-0.0181\end{array}$ & $\begin{array}{l}\text { BTC-Tencent } \\
-0.0025\end{array}$ & $\begin{array}{l}\text { BTC-JD } \\
-0.0049\end{array}$ & $\begin{array}{c}\text { BTC-Alibaba } \\
-0.0091\end{array}$ & $\begin{array}{c}\sum \text { Pairwise spillovers } \\
\quad=-0.1873\end{array}$ \\
\hline \multicolumn{5}{|c|}{ Spillovers to BTC* } \\
\hline IBM & Intel & BofA & Mastercard & Visa \\
\hline 0.0160 & 0.1432 & 0.0470 & 0.2212 & 0.1146 \\
\hline Microsoft & Walmart & $\mathrm{BT}$ & Accenture & Ping An \\
\hline 0.2536 & NA & NA & 0.0485 & NA \\
\hline Baidu & Tencent & JD & Alibaba & \\
\hline 0.0817 & 0.0113 & 0.0221 & 0.0409 & \\
\hline
\end{tabular}

Note: The top 3 contributors to the volatility of Bitcoin are highlighted in bold. *Spillovers to Bitcoin are calculated as: $\frac{\text { Negative pairwise spillovers }}{\sum \text { Negative pairwise spillovers }}$ 
Table 4: Pairwise spillovers using the approach developed by Baruník and Křehlík [2018] (BK18)

\begin{tabular}{|c|c|c|c|c|c|}
\hline \multicolumn{6}{|c|}{ Pairwise spillovers using BK18 in \% } \\
\hline $\begin{array}{l}\text { Freq. } 1 \\
\text { (1-4 days) }\end{array}$ & $\begin{array}{c}\text { BTC-IBM } \\
-0.0050 \\
\text { BTC-Microsoft } \\
-0.0091 \\
\text { BTC-Baidu } \\
-0.0044\end{array}$ & $\begin{array}{c}\text { BTC-Intel } \\
-0.0037 \\
\text { BTC-Walmart } \\
-0.0039 \\
\text { BTC-Tencent } \\
-0.0012\end{array}$ & $\begin{array}{c}\text { BTC-BofA } \\
-0.0048 \\
\text { BTC-BT } \\
0.0079 \\
\text { BTC-JD } \\
-0.0007\end{array}$ & $\begin{array}{c}\text { BTC-Mastercard } \\
-0.0087 \\
\text { BTC-Accenture } \\
-0.0076 \\
\text { BTC-Alibaba } \\
-0.0020\end{array}$ & $\begin{array}{c}\text { BTC-Visa } \\
-0.0108 \\
\text { BTC-Ping An } \\
0.0016 \\
\sum \text { Pairwise spillovers } \\
=-0.0524\end{array}$ \\
\hline Spillovers to BTC* & $\begin{array}{c}\text { IBM } \\
0.0809 \\
\text { Microsoft } \\
\mathbf{0 . 1 4 6 6} \\
\text { Baidu } \\
0.0712 \\
\end{array}$ & $\begin{array}{c}\text { Intel } \\
0.0599 \\
\text { Walmart } \\
0.0633 \\
\text { Tencent } \\
0.0195 \\
\end{array}$ & $\begin{array}{l}\text { BofA } \\
0.0772 \\
\text { BT } \\
\text { NA } \\
\text { JD } \\
0.0110 \\
\end{array}$ & $\begin{array}{c}\text { Mastercard } \\
\mathbf{0 . 1 3 9 7} \\
\text { Accenture } \\
0.1232 \\
\text { Alibaba } \\
0.0325 \\
\end{array}$ & $\begin{array}{c}\text { Visa } \\
\mathbf{0 . 1 7 5 0} \\
\text { Ping An } \\
\text { NA }\end{array}$ \\
\hline $\begin{array}{l}\text { Freq. } 2 \\
\text { (5-10 days) }\end{array}$ & $\begin{array}{c}\text { BTC-IBM } \\
-0.0019 \\
\text { BTC-Microsoft } \\
-0.0240 \\
\text { BTC-Baidu } \\
-0.0092 \\
\end{array}$ & $\begin{array}{c}\text { BTC-Intel } \\
-0.0121 \\
\text { BTC-Walmart } \\
0.0034 \\
\text { BTC-Tencent } \\
-0.0028 \\
\end{array}$ & $\begin{array}{c}\text { BTC-BofA } \\
-0.0050 \\
\text { BTC-BT } \\
0.0007 \\
\text { BTC-JD } \\
-0.0021 \\
\end{array}$ & $\begin{array}{c}\text { BTC-Mastercard } \\
-0.0206 \\
\text { BTC-Accenture } \\
-0.0042 \\
\text { BTC-Alibaba } \\
-0.0039 \\
\end{array}$ & $\begin{array}{c}\text { BTC-Visa } \\
-0.0100 \\
\text { BTC-Ping An } \\
0.0037 \\
\sum \text { Pairwise spillovers } \\
=-0.0880 \\
\end{array}$ \\
\hline Spillovers to BTC* & $\begin{array}{c}\text { IBM } \\
0.0194 \\
\text { Microsoft } \\
\mathbf{0 . 2 5 0 8} \\
\text { Baidu } \\
0.0956 \\
\end{array}$ & $\begin{array}{c}\text { Intel } \\
\mathbf{0 . 1 2 6 5} \\
\text { Walmart } \\
\text { NA } \\
\text { Tencent } \\
0.0293 \\
\end{array}$ & $\begin{array}{c}\text { BofA } \\
0.0523 \\
\text { BT } \\
\text { NA } \\
\text { JD } \\
0.0226 \\
\end{array}$ & $\begin{array}{c}\text { Mastercard } \\
\mathbf{0 . 2 1 5 0} \\
\text { Accenture } \\
0.0440 \\
\text { Alibaba } \\
0.0407 \\
\end{array}$ & $\begin{array}{c}\text { Visa } \\
0.1039 \\
\text { Ping An } \\
\text { NA }\end{array}$ \\
\hline $\begin{array}{l}\text { Freq. } 3 \\
(>10 \text { days })\end{array}$ & $\begin{array}{c}\text { BTC-IBM } \\
0.0033 \\
\text { BTC-Microsoft } \\
-0.0233 \\
\text { BTC-Baidu } \\
-0.0046\end{array}$ & $\begin{array}{c}\text { BTC-Intel } \\
-0.0160 \\
\text { BTC-Walmart } \\
0.0087 \\
\text { BTC-Tencent } \\
0.0015\end{array}$ & $\begin{array}{c}\text { BTC-BofA } \\
-0.0007 \\
\text { BTC-BT } \\
-0.0017 \\
\text { BTC-JD } \\
-0.0021\end{array}$ & $\begin{array}{c}\text { BTC-Mastercard } \\
-0.0199 \\
\text { BTC-Accenture } \\
0.0011 \\
\text { BTC-Alibaba } \\
-0.0032\end{array}$ & $\begin{array}{c}\text { BTC-Visa } \\
-0.0047 \\
\text { BTC-Ping An } \\
0.0145 \\
\sum \text { Pairwise spillovers } \\
=-0.0470\end{array}$ \\
\hline Spillovers to BTC* & $\begin{array}{c}\text { IBM } \\
\text { NA } \\
\text { Microsoft } \\
\mathbf{0 . 3 0 6 2} \\
\text { Baidu } \\
0.0601 \\
\end{array}$ & $\begin{array}{c}\text { Intel } \\
\mathbf{0 . 2 1 0 4} \\
\text { Walmart } \\
\text { NA } \\
\text { Tencent } \\
\text { NA } \\
\end{array}$ & $\begin{array}{c}\text { BofA } \\
0.0087 \\
\text { BT } \\
0.0221 \\
\text { JD } \\
0.0272 \\
\end{array}$ & $\begin{array}{c}\text { Mastercard } \\
\mathbf{0 . 2 6 2 1} \\
\text { Accenture } \\
\text { NA } \\
\text { Alibaba } \\
0.0417 \\
\end{array}$ & $\begin{array}{c}\text { Visa } \\
0.0615 \\
\text { Ping An } \\
\text { NA }\end{array}$ \\
\hline
\end{tabular}

Note: The top 3 contributors to the volatility of Bitcoin are highlighted in bold. *Spillovers to Bitcoin are calculated as: Negative pairwise spillovers

$\sum$ Negative pairwise spillovers

Table 5: Rolling window estimates for net volatility spillovers of Bitcoin.

\begin{tabular}{lc|ccc}
\hline \multicolumn{3}{c}{ Rolling window estimates for net volatility spillovers of Bitcoin in \% } \\
\hline & DY12 & \multicolumn{4}{c}{ BK18 } \\
\hline & & $\begin{array}{c}\text { Freq. 1 } \\
(1-4 \text { days })\end{array}$ & $\begin{array}{c}\text { Freq. } 2 \\
(5-10 \text { days })\end{array}$ & $\begin{array}{c}\text { Freq. 3 } \\
(>10 \text { days })\end{array}$ \\
\hline Max & 0.9852 & 0.1254 & 0.0874 & 0.1931 \\
Min & -2.4039 & -0.5572 & -0.7742 & -2.0648 \\
Mean & -0.7578 & -0.1422 & -0.2068 & -0.2850 \\
S.D. & 0.4624 & 0.1257 & 0.1918 & 0.3381 \\
\hline \hline
\end{tabular}

Note: The above table presents the rolling window estimates for pairwise spillovers between Bitcoin (BTC) and the stated blockchain patent-related stocks across multiple time frequencies using the approach developed by both Diebold and Yilmaz [2012] (DY12) and Baruník and Křehlík [2018] (BK18). 
Table 6: Rolling window estimates for pairwise spillovers between Bitcoin and blockchain patent-related stocks.

\begin{tabular}{|c|c|c|c|c|c|}
\hline \multicolumn{6}{|c|}{ Rolling window estimates for pairwise spillovers in \% } \\
\hline & & DY12 & \multicolumn{3}{|c|}{ BK18 } \\
\hline & & & $\begin{array}{c}\text { Freq. } 1 \\
\text { (1-4 days) }\end{array}$ & $\begin{array}{c}\text { Freq. } 2 \\
\text { (5-10 days) }\end{array}$ & $\begin{array}{c}\text { Freq. } 3 \\
(>10 \text { days })\end{array}$ \\
\hline \multirow[t]{4}{*}{ IBM } & Max & 0.1827 & 0.0205 & 0.0223 & 0.0442 \\
\hline & Min & -0.3510 & -0.0971 & -0.0680 & -0.1927 \\
\hline & Mean & -0.0415 & -0.0170 & -0.0090 & -0.0113 \\
\hline & S.D. & 0.0596 & 0.0239 & 0.0152 & 0.0264 \\
\hline \multirow[t]{4}{*}{ Intel } & Max & 0.0787 & 0.0191 & 0.0168 & 0.0259 \\
\hline & Min & -0.5006 & -0.0919 & -0.1377 & -0.3248 \\
\hline & Mean & -0.0854 & -0.0117 & -0.0194 & -0.0271 \\
\hline & S.D. & 0.0992 & 0.0160 & 0.0248 & 0.0419 \\
\hline \multirow[t]{4}{*}{ BofA } & $\operatorname{Max}$ & 0.1410 & 0.0467 & 0.0234 & 0.0351 \\
\hline & Min & -0.5803 & -0.0561 & -0.0775 & -0.2230 \\
\hline & Mean & -0.0719 & -0.0086 & -0.0141 & -0.0224 \\
\hline & S.D. & 0.0972 & 0.0136 & 0.0220 & 0.0391 \\
\hline \multirow[t]{4}{*}{ Mastercard } & $\operatorname{Max}$ & 0.1294 & 0.0327 & 0.0254 & 0.0270 \\
\hline & Min & -0.6457 & -0.1637 & -0.1303 & -0.3498 \\
\hline & Mean & -0.1493 & -0.0299 & -0.0414 & -0.0528 \\
\hline & S.D. & 0.1671 & 0.0432 & 0.0454 & 0.0646 \\
\hline \multirow[t]{4}{*}{ Visa } & $\operatorname{Max}$ & 0.1458 & 0.0706 & 0.0295 & 0.0219 \\
\hline & Min & -0.3992 & -0.1113 & -0.1722 & -0.2976 \\
\hline & Mean & -0.0995 & -0.0232 & -0.0362 & -0.0464 \\
\hline & S.D. & 0.1064 & 0.0373 & 0.0404 & 0.0577 \\
\hline \multirow[t]{4}{*}{ Microsoft } & $\operatorname{Max}$ & 0.0965 & 0.0066 & 0.0084 & 0.0069 \\
\hline & Min & -0.4045 & -0.1348 & -0.1031 & -0.2930 \\
\hline & Mean & -0.1331 & -0.0245 & -0.0306 & -0.0380 \\
\hline & S.D. & 0.0953 & 0.0249 & 0.0292 & 0.0415 \\
\hline \multirow[t]{4}{*}{ Walmart } & $\operatorname{Max}$ & 0.2729 & 0.0279 & 0.0567 & 0.0868 \\
\hline & Min & -0.1932 & -0.1027 & -0.0880 & -0.1715 \\
\hline & Mean & -0.0374 & -0.0096 & -0.0062 & -0.0060 \\
\hline & S.D. & 0.0598 & 0.0221 & 0.0193 & 0.0235 \\
\hline
\end{tabular}

Note: The above table presents the rolling window estimates for pairwise spillovers between Bitcoin (BTC) and the stated blockchain patent-related stocks across multiple time frequencies using the approach developed by both Diebold and Yilmaz [2012] (DY12) and Baruník and Křehlík [2018] (BK18). 
Table 7: Rolling window estimates for pairwise spillovers between Bitcoin and blockchain patent-related stocks.

\begin{tabular}{|c|c|c|c|c|c|}
\hline \multicolumn{6}{|c|}{ Rolling window estimates for pairwise spillovers in \% } \\
\hline & & DY12 & & BK18 & \\
\hline & & & $\begin{array}{c}\text { Freq. } 1 \\
\text { (1-4 days) }\end{array}$ & $\begin{array}{c}\text { Freq. } 2 \\
\text { (5-10 days) }\end{array}$ & $\begin{array}{c}\text { Freq. } 3 \\
(>10 \text { days })\end{array}$ \\
\hline \multirow[t]{4}{*}{$\overline{\mathrm{BT}}$} & Max & 0.2457 & 0.0633 & 0.0783 & 0.0797 \\
\hline & Min & -0.2401 & -0.0477 & -0.0457 & -0.0955 \\
\hline & Mean & 0.0304 & 0.0018 & -0.0023 & -0.0065 \\
\hline & S.D. & 0.0617 & 0.0151 & 0.0165 & 0.0239 \\
\hline \multirow[t]{4}{*}{ Accenture } & $\operatorname{Max}$ & 0.0495 & 0.0165 & 0.0107 & 0.0125 \\
\hline & Min & -0.2663 & -0.0781 & -0.1662 & -0.2102 \\
\hline & Mean & -0.0717 & -0.0168 & -0.0239 & -0.0279 \\
\hline & S.D. & 0.0595 & 0.0176 & 0.0275 & 0.0310 \\
\hline \multirow[t]{4}{*}{ Ping An } & Max & 0.4513 & 0.1833 & 0.1729 & 0.1847 \\
\hline & Min & -0.3135 & -0.0368 & -0.0798 & -0.0805 \\
\hline & Mean & 0.0664 & 0.0227 & 0.0312 & 0.0298 \\
\hline & S.D. & 0.1630 & 0.0364 & 0.0511 & 0.0517 \\
\hline \multirow[t]{4}{*}{ Baidu } & Max & 0.0671 & 0.0218 & 0.0220 & 0.0301 \\
\hline & Min & -0.4988 & -0.0814 & -0.2470 & -0.3129 \\
\hline & Mean & -0.0711 & -0.0158 & -0.0271 & -0.0332 \\
\hline & S.D. & 0.1142 & 0.0213 & 0.0583 & 0.0762 \\
\hline \multirow[t]{4}{*}{ Tencent } & Max & 0.2326 & 0.0609 & 0.0391 & 0.0632 \\
\hline & Min & -0.4160 & -0.0372 & -0.0510 & -0.0694 \\
\hline & Mean & -0.0255 & 0.0040 & -0.0022 & -0.0043 \\
\hline & S.D. & 0.0769 & 0.0121 & 0.0151 & 0.0196 \\
\hline \multirow[t]{4}{*}{ JD } & Max & 0.3719 & 0.0260 & 0.0232 & 0.0382 \\
\hline & Min & -0.2371 & -0.0567 & -0.0630 & -0.1276 \\
\hline & Mean & -0.0308 & -0.0058 & -0.0112 & -0.0161 \\
\hline & S.D. & 0.0625 & 0.0138 & 0.0193 & 0.0286 \\
\hline \multirow[t]{4}{*}{ Alibaba } & $\operatorname{Max}$ & 0.2291 & 0.0127 & 0.0136 & 0.0223 \\
\hline & Min & -0.2111 & -0.0587 & -0.0915 & -0.1536 \\
\hline & Mean & -0.0374 & -0.0080 & -0.0146 & -0.0228 \\
\hline & S.D. & 0.0509 & 0.0140 & 0.0211 & 0.0337 \\
\hline
\end{tabular}

Note: The above table presents the rolling window estimates for pairwise spillovers between Bitcoin (BTC) and the stated blockchain patent-related stocks across multiple time frequencies using the approach developed by both Diebold and Yilmaz [2012] (DY12) and Baruník and Křehlík [2018] (BK18). 